\title{
Thermodynamical second-order hydrodynamic coefficients
}

\author{
Guy D. Moore and Kiyoumars A. Sohrabi \\ Physics Department, McGill University, 3600 rue University, Montréal, QC H3A 2T8, \\ Canada
}

\begin{abstract}
Transport coefficients in non-conformal second-order hydrodynamics can be classified as either dynamical or thermodynamical. We derive Kubo formuale for the thermodynamical coefficients and compute them at leading perturbative order in a theory with general matter content. We also discuss how to approach their evaluation on the lattice.
\end{abstract}




\section{Contents}

1 Introduction 1

2 Hydrodynamics 3

$\begin{array}{lll}3 & \text { Evaluation at Weak Coupling } & 6\end{array}$

3.1 Scalars 8

$\begin{array}{ll}3.2 \text { Gauge fields } & 11\end{array}$

$\begin{array}{lll}3.3 & \text { Fermions } & 12\end{array}$

$\begin{array}{lll}3.4 & \text { Results } & 13\end{array}$

4 Lattice implementation $\quad 14$

$\begin{array}{llr}5 & \text { Discussion } & 17\end{array}$

$\begin{array}{lr}\text { A Non-conformal hydrodynamics } & 17\end{array}$

$\begin{array}{lll}\text { A.1 Kubo relation for } \kappa \text { and } \xi_{5} & 19\end{array}$

A.2 Kubo relation for $\kappa^{*}$ and $\xi_{6} \quad 21$

A.3 Kubo relation for $\lambda_{3}$ and $\xi_{3} \quad 22$

A.4 Kubo relation for $\lambda_{4}$ and $\xi_{4} \quad 24$

\section{Introduction}

The theory of QCD is weakly coupled at short distances or high temperatures, but strongly coupled at long distances or low temperatures. One of the major goals of both the experimental and theoretical programs in QCD has been to understand how quickly this transition occurs and at what energy. A major purpose of the heavy ion collision program was to see if weak-coupling behavior emerges at available energies. Similarly, lattice studies have investigated how close thermodynamic properties come to their weak-coupling values as a function of the temperature.

Broadly speaking, we can divide properties of thermal QCD into two categories: dynamical and thermodynamical. Most of our information on dynamical properties is from experiment. Experiments show [1] that at available temperatures, QCD displays excellent fluid behavior with remarkably low viscosity [2]. This is very different from weak-coupling behavior [3, 4], but roughly consistent with strong-coupling behavior in similar theories which we can solve $[5,6]$. The story for thermodynamic properties, where most of our information is from the lattice, is more complex. At $T \sim 150$ to 200 
$\mathrm{MeV}$, thermodynamic properties such as pressure, baryon susceptibility, and $\langle\bar{\psi} \psi\rangle$ show strong temperature dependence and are far from their weak-coupling values [7]. As temperature rises most thermodynamic quantities approach weak-coupling behavior, but at different rates. Quark number susceptibilities come close to weakcoupling behavior already at a few $T_{c}[7,8]$. Cross-correlations between strange and light quark numbers transition change from the expected behavior in a hadron gas to the behavior of a weakly coupled plasma over this same temperature range. The pressure takes rather longer to approach weak coupling behavior [9].

We feel that the more dynamical and thermodynamical quantities we have available, the more complete and nuanced a picture of the strong to weak coupling transition we can obtain. With this in mind, we advocate investigating the so-called second order hydrodynamic coefficients and their coupling dependence. As we will argue, some of these coefficients are thermodynamical and can be computed on the lattice. They also have simple weak-coupling behavior. Indeed, the main goal of this paper will be to compute their leading-order weak-coupling behavior in a general theory. We will also discuss what would be involved in evaluating them on the lattice.

In the the next section we will review second-order hydrodynamics and explain how some of the coefficients of this theory are thermodynamical. Since at least the mid-rapidity regions in high-energy heavy ion collisions deal with QCD at small quark-number chemical potentials, and since the lattice can only deal well with the case where chemical potentials vanish, we will assume vanishing quark-number chemical potentials. We also ignore magnetic fields. However, QCD is far from a conformal theory in the interesting energy regime, so we will not assume conformal symmetry. In this case there are three independent second-order hydrodynamic coefficients which are thermodynamical in nature $[10,12]$. In the notation of Romatschke $[13,14]$ these are $\kappa, \lambda_{3}$ and $\lambda_{4}$. We compute their values at weak coupling and vanishing masses in Section 3; see particularly Eq. (3.38) and Eq. (3.39).

As we remarked, it would be interesting to evaluate these coefficients for QCD on the lattice. This can be done because these coefficients all have Kubo relations directly in terms of finite-temperature, Euclidean correlation functions of the sort which can be evaluated on the lattice (without the need for analytic continuation). This is possible precisely because these transport coefficients are thermodynamical in nature. Explicit expressions for the Kubo relations are found in the next section, see Eq. (2.10) to Eq. (2.12). In Section 4, we present a brief discussion of how these Kubo relations might be applied on the lattice. In particular, we discuss operator normalization and contact terms. Based on this discussion, we think the evaluation of $\kappa$ should be feasible with existing techniques, at least for pure-glue QCD [15]. The evaluation of $\lambda_{3}$ and $\lambda_{4}$ may be prohibitively difficult since computation of three-point functions are always much harder than two-point functions on lattice. 


\section{Hydrodynamics}

Hydrodynamics is a general theoretical framework for describing the behavior of fluids locally near equilibrium. It is organized as an expansion in gradients of the fluid properties. (For a recent review of relativistic hydrodynamics see $[16,17]$ ). At lowest (zero) order in gradients, hydrodynamics is determined by equilibrium thermodynamics. The state of the fluid at each point is determined by the values of all conserved charge densities. For QCD, these are the momentum density $\mathcal{P}^{\mu}$ associated with the stress tensor $T^{\mu \nu}$ and the charge densities $Q_{a}, a=u, d, s, \ldots$ associated with the conserved 4-currents $J_{a}^{\mu}$. Assuming local equilibrium and an equation of state for the pressure $P$ in terms of the energy density and charge densities, $P=P\left(\epsilon, n_{a}\right)$, these currents can be determined in terms of the conserved charge densities. In practice one uses a slightly more convenient set of variables; ${ }^{1}$ the energy density and flow 4-velocity, $\mathcal{P}^{\mu}=\epsilon u^{\mu}$ with $g_{\mu \nu} u^{\mu} u^{\nu}=-1$ (where $g_{\mu \nu}$ is the metric tensor, in flat space $\left.g_{\mu \nu}=\eta_{\mu \nu}=\operatorname{Diag}[-1,1,1,1]\right)$ and the number densities $n_{a} \equiv g_{\mu \nu} u^{\mu} J_{a}^{\nu}$. In terms of these the stress tensor and current at lowest order are

$$
\begin{aligned}
T^{\mu \nu}(\epsilon, u, n) & =(\epsilon+P) u^{\mu} u^{\nu}+P g^{\mu \nu}=\epsilon u^{\mu} u^{\nu}+P \Delta^{\mu \nu} \\
J_{a}^{\mu}(\epsilon, u, n) & =u^{\mu} n_{a} .
\end{aligned}
$$

Here $\Delta^{\mu \nu} \equiv g^{\mu \nu}+u^{\mu} u^{\nu}$ is a projection operator onto the local spatial directions. When $T^{\mu \nu}$ and $J^{\mu}$ satisfy these expressions, then stress conservation, $\nabla_{\mu} T^{\mu \nu}$ and current conservation, $\nabla_{\mu} J_{a}^{\mu}$, close and completely determine the fluid dynamics. The relevant quantities at this order - the pressure $P$ and its various derivatives which give the entropy density $s$, quark number susceptibilities $\chi_{a b}$, the speed of sound $c_{s}$, and so forth - have been extensively studied on the lattice $[7,8]$.

At first order in gradients there are two independent terms one can add to the righthand side of Eq. (2.1):

$$
\begin{aligned}
& T^{\mu \nu}=\text { RHS of Eq. }(2.1)-\eta \sigma^{\mu \nu}-\zeta \Delta^{\mu \nu} \nabla_{\alpha} u^{\alpha}, \\
& \sigma^{\mu \nu} \equiv \Delta^{\mu \alpha} \Delta^{\nu \beta}\left(\nabla_{\alpha} u_{\beta}+\nabla_{\beta} u_{\alpha}-\frac{2}{3} \Delta_{\alpha \beta} \nabla_{\gamma} u^{\gamma}\right) .
\end{aligned}
$$

Here $\eta, \zeta$ are the shear and bulk viscosities respectively. These new coefficients $\eta$, $\zeta$, and the diffusion coefficient which can be added to Eq. (2.2), are all dynamical quantities. If the coefficients and the terms they multiply are nonzero then entropy increases. They can be determined, via Kubo relations, from equilibrium correlation functions of stress tensor or current operators, but the relations involve evaluating these correlation functions at nonzero frequency, which makes a direct evaluation on the lattice impossible and an indirect evaluation at best very challenging [18-20]. However there is significant progress in determining them from experiment [21].

\footnotetext{
${ }^{1}$ Note that we are using the Landau-Lifshitz frame.
} 
At second order there are a host of terms which can be added. The situation improves somewhat if we assume that charge densities are small, so $J_{a}^{\mu}$ terms can be neglected. If in addition we assume that the theory under consideration is conformally invariant, then there are 5 additional terms which must be included [14]. However, since we are interested in QCD at finite coupling and potentially in making contact with the lattice, we cannot assume conformal invariance. In this case, after reducing the number of terms by applying equations of motion and other interrelations, there are 15 independent terms which appear at second order, which have been enumerated by Romatschke [13]. To write these terms explicitly it is convenient to introduce the vorticity tensor $\Omega^{\mu \nu}$,

$$
2 \Omega^{\mu \nu} \equiv \Delta^{\mu \alpha} \Delta^{\nu \beta}\left(\nabla_{\alpha} u_{\beta}-\nabla_{\beta} u_{\alpha}\right)
$$

as well as the curvature tensor $R^{\mu \nu \alpha \beta}$ and Ricci tensor $R^{\mu \nu}=R_{\alpha}^{\mu}{ }^{\nu \alpha}$ and scalar $R=R_{\mu}^{\mu}$. And we will write

$$
R^{\mu\langle\nu \alpha\rangle \beta} \equiv \frac{1}{2} R^{\mu \kappa \sigma \beta}\left(\Delta_{\kappa}^{\nu} \Delta_{\sigma}^{\alpha}+\Delta_{\sigma}^{\nu} \Delta_{\kappa}^{\alpha}-\frac{2}{3} \Delta^{\nu \alpha} \Delta_{\kappa \sigma}\right)
$$

and similarly for $R^{\langle\mu \nu\rangle}$. That is, the indices enclosed in angle brackets are spaceprojected, symmetrized, and trace-subtracted. Using all of this notation, the possible second-order terms, according to Romatschke [13], are

$$
\begin{aligned}
T^{\mu \nu}= & \text { Eq. }(2.3)+\eta \tau_{\pi}\left(u \cdot \nabla \sigma^{\mu \nu}+\frac{\nabla \cdot u}{3} \sigma^{\mu \nu}\right) \\
& +\kappa\left(R^{\langle\mu \nu\rangle}-2 u_{\alpha} u_{\beta} R^{\alpha\langle\mu \nu\rangle \beta}\right) \\
& +\lambda_{1} \sigma_{\lambda}{ }^{\langle\mu} \sigma^{\nu\rangle \lambda}+\lambda_{2} \sigma_{\lambda}{ }^{\langle\mu} \Omega^{\nu\rangle \lambda}-\lambda_{3} \Omega_{\lambda}{ }^{\langle\mu} \Omega^{\nu\rangle \lambda} \\
& +\eta \tau_{\pi}^{*} \frac{\nabla \cdot u}{3} \sigma^{\mu \nu}+\lambda_{4} \nabla^{\langle\mu} \ln s \nabla^{\nu\rangle} \ln s+2 \kappa^{*} u_{\alpha} u_{\beta} R^{\alpha\langle\mu \nu\rangle \beta} \\
& +\Delta^{\mu \nu}\left(-\zeta \tau_{\Pi} u \cdot \nabla \nabla \cdot u+\xi_{1} \sigma^{\alpha \beta} \sigma_{\alpha \beta}+\xi_{2}(\nabla \cdot u)^{2}\right. \\
& \left.\quad+\xi_{4} \nabla_{\alpha \perp} \ln s \nabla_{\perp}^{\alpha} \ln s+\xi_{3} \Omega^{\alpha \beta} \Omega_{\alpha \beta}+\xi_{5} R+\xi_{6} u^{\alpha} u^{\beta} R_{\alpha \beta}\right) .
\end{aligned}
$$

There are several ways to categorize these terms. Some are only relevant in curved space; $\kappa, \kappa^{*}, \xi_{5}$ and $\xi_{6}$. The others are relevant in flat or curved space. (Even though $\kappa$ etc. only play a role in curved space, they mix with the other terms when we find Kubo relations in Eqs. (2.10-2.12), so they should generally be considered anyway [14].) We can also divide the terms into linear and nonlinear terms. Linear terms affect small fluctuations and can, for instance, influence their dispersion; nonlinear terms are only relevant at second order in small fluctuations about equilibrium and flat space. The linear terms are $\tau_{\pi}, \kappa, \kappa^{*}, \tau_{\Pi}, \xi_{5}$, and $\xi_{6}$. The other terms, $\lambda_{1 \ldots 4}, \tau_{\pi}^{*}$, $\xi_{1 \ldots 4}$ are nonlinear.

We can also group these terms into those which are thermodynamical in nature, and those which are dynamical. We call a term thermodynamical if it can give a 
nonzero contribution to $T^{\mu \nu}$ when the geometry and density matrix are fully timeindependent and the system is therefore in equilibrium. No term involving the shear tensor $\sigma_{\mu \nu}$ is thermodynamical because a system under shear flow is changing with time and is producing entropy. In nonconformal theories, the same is true of bulk flow $\nabla \cdot u$. However, it is completely consistent to have a system which is in equilibrium in a curved (but time-independent) geometry. Similarly, a time-independent but spacevarying $g_{00}$ (gravitational potential) makes $\nabla_{\mu \perp} s$ nonzero without any departure from equilibrium. Similarly, it is possible (in a curved geometry) to establish persistent vorticity which is sustained forever. ${ }^{2}$ The system will be fully in equilibrium in the presence of this vorticity. Hence, the coefficients $\kappa, \kappa^{*}, \lambda_{3}, \lambda_{4}, \xi_{3}, \xi_{4}, \xi_{5}, \xi_{6}$ represent thermodynamical quantities.

In Ref. [22] we showed how to derive Kubo relations for second-order hydro coefficients. There we did so only for conformal theories, but it is straightforward to do so for nonconformal theories as well. Doing so, we find that the Kubo relations for the thermodynamical coefficients can all be expressed in terms of retarded correlation functions evaluated directly at zero frequency. Up to powers of $i$, zero-frequency retarded correlators equal zero-frequency Euclidean correlators. In fact, we can derive (Kubo) relations between the thermodynamic coefficients and Euclidean correlators by working directly in Euclidean space. In particular, defining the Euclidean $n$-point function as

$$
\begin{aligned}
G_{\mathrm{E}}^{\mu_{1} \nu_{1} \ldots \mu_{n} \nu_{n}}\left(p_{1}, \ldots, p_{n-1},-p_{1}-\ldots-p_{n-1}\right) \equiv & \int d^{4} x_{1} \ldots d^{4} x_{n-1} e^{-i\left(p_{1} \cdot x_{1}+\ldots+p_{n-1} \cdot x_{n-1}\right)} \\
& \times\left.\frac{2^{n} \partial^{n} \ln \mathrm{Z}}{\partial g_{\mu_{1} \nu_{1}}\left(x_{1}\right) \ldots \partial g_{\mu_{n} \nu_{n}}(0)}\right|_{g_{\mu \nu}=\delta_{\mu \nu}}
\end{aligned}
$$

with

$$
Z\left[g_{\mu \nu}\right]=\int \mathcal{D} \phi \exp \left\{-S_{\mathrm{E}}\left[\phi, g_{\mu \nu}\right]\right\}
$$

we find

$$
\begin{aligned}
\kappa & =\left.\lim _{k_{z} \rightarrow 0} \frac{\partial^{2}}{\partial k_{z}^{2}} G_{E}^{x y, x y}(k)\right|_{k_{0}=0}, \\
\lambda_{3} & =2 \kappa^{*}-\left.4 \lim _{p^{z}, q^{z} \rightarrow 0} \frac{\partial^{2}}{\partial p_{z} \partial q_{z}} G_{E}^{x t, y t, x y}(p, q)\right|_{p_{0}, q_{0}=0}, \\
\lambda_{4} & =-2 \kappa^{*}+\kappa-\left.\frac{c_{s}^{4}}{2} \lim _{p^{x}, q^{y} \rightarrow 0} \frac{\partial^{2}}{\partial p_{x} \partial q_{y}} G_{E}^{t t, t t, x y}(p, q)\right|_{p_{0}, q_{0}=0} .
\end{aligned}
$$

The remaining transport coefficients, including $\kappa^{*}$ which appears above, are not independent but are determined in terms of these three via five independent conditions.

\footnotetext{
${ }^{2}$ For instance, consider a spacetime which is $\mathcal{S}^{2} \times \mathcal{R}^{2}$, with time in one of the flat directions. The fluid can spin about the equator of the $\mathcal{S}^{2}$ and this flow will persist forever, and will therefore reach equilibrium.
} 
Two conditions were found by Romatschke [16], by demanding that the entropy current have non-negative divergence. His calculation was limited to second order in gradients; but a treatment to third order in gradients by Bhattacharyya [10] and Jensen et al [11] found three more constraints on second order transport coefficients. For an interesting physical interpretation of these constraints, see [12].

The five constraints found by Bhattacharyya, in our notation ${ }^{3}$, are

$$
\begin{aligned}
\kappa^{*}= & \kappa-\frac{T}{2} \frac{d \kappa}{d T}, \\
\xi_{5}= & \frac{1}{2}\left(c_{s}^{2} T \frac{d \kappa}{d T}-c_{s}^{2} \kappa-\frac{\kappa}{3}\right) \\
\xi_{6}= & c_{s}^{2}\left(3 T \frac{d \kappa}{d T}-2 T \frac{d \kappa^{*}}{d T}+2 \kappa^{*}-3 \kappa\right)-\kappa+\frac{4 \kappa^{*}}{3}+\frac{\lambda_{4}}{c_{s}^{2}} \\
\xi_{3}= & \frac{3 c_{s}^{2} T}{2}\left(\frac{d \kappa^{*}}{d T}-\frac{d \kappa}{d T}\right)+\frac{3\left(c_{s}^{2}-1\right)}{2}\left(\kappa^{*}-\kappa\right)-\frac{\lambda_{4}}{c_{s}^{2}}+\frac{1}{4}\left(c_{s}^{2} T \frac{d \lambda_{3}}{d T}-3 c_{s}^{2} \lambda_{3}+\frac{\lambda_{3}}{3}\right), \\
\xi_{4}= & -\frac{\lambda_{4}}{6}-\frac{c_{s}^{2}}{2}\left(\lambda_{4}+T \frac{d \lambda_{4}}{d T}\right)+c_{s}^{4}\left(1-3 c_{s}^{2}\right)\left(T \frac{d \kappa}{d T}-T \frac{d \kappa^{*}}{d T}+\kappa^{*}-\kappa\right) \\
& -c_{s}^{6} T^{3} \frac{d^{2}}{d T^{2}}\left(\frac{\kappa-\kappa^{*}}{T}\right) .
\end{aligned}
$$

We take these constraints to determine all other coefficients in terms of $\kappa, \lambda_{3}$, and $\lambda_{4}$. In Appendix A we give a detailed derivation of Eqs.(2.10-2.12), and we find Kubo relations for the dependent transport coefficients mentioned in Eqs. (2.13-2.17) for completeness.

Euclidean correlation functions have well behaved perturbative expansions at finite temperature (at least at low order), therefore it should be possible to evaluate these correlators perturbatively in a weakly coupled theory. We present the derivation at lowest order in a general massless theory for particles of spin zero, half and one in the next section.

\section{Evaluation at Weak Coupling}

To carry out the calculation of these transport coefficients, first we have to clarify the nature of the correlation functions that are derived by differentiating the curved space partition function. The definition for the $n$-point Green function established in Eq. (2.8) involves multiple derivatives acting on the energy functional. Each

\footnotetext{
${ }^{3}$ Labeling the coefficients of [12] with a prime, the relations between their coefficients $\kappa_{1}^{\prime}, \kappa_{2}^{\prime}, \lambda_{3}^{\prime}$, $\lambda_{4}^{\prime}, \zeta_{2}^{\prime}, \zeta_{3}^{\prime}, \xi_{3}^{\prime}$, and $\xi_{4}^{\prime}$ and our coefficients are: $T \kappa_{1}^{\prime}=\kappa, T \kappa_{2}^{\prime}=2 \kappa-2 \kappa^{*},-T \lambda_{3}^{\prime}=\lambda_{3}, c_{s}^{4} T \lambda_{4}^{\prime}=\lambda_{4}$, $T \zeta_{2}^{\prime}=\xi_{5}, T \zeta_{3}^{\prime}=\xi_{6},-T \xi_{3}^{\prime}=\xi_{3}$ and $c_{s}^{4} T \xi_{4}^{\prime}=\xi_{4}$. Moreover, unlike [12] our convention for $R^{\rho \sigma \mu \nu}$ is $R_{\sigma \mu \nu}^{\rho}=\partial_{\mu} \Gamma_{\nu \sigma}^{\rho}-\partial_{\nu} \Gamma_{\mu \sigma}^{\rho}+\Gamma_{\mu \lambda}^{\rho} \Gamma_{\nu \sigma}^{\lambda}-\Gamma_{\nu \lambda}^{\rho} \Gamma_{\mu \sigma}^{\lambda}$.
} 
derivative can pull down a factor of $-2 \partial \mathcal{L}_{\mathrm{E}} / \partial g_{\mu \nu}=T^{\mu \nu}$, giving a conventional $n$ point stress-tensor correlator; but the $g_{\mu \nu}$ derivatives can also act on $T^{\alpha \beta}$ factors pulled down by previous $g_{\alpha \beta}$ derivatives, leading to contact terms. (In intermediate steps our $T^{\mu \nu}$ is really the stress tensor density $\sqrt{g} T^{\mu \nu}$; the distinction is irrelevant in final expressions since in the end we evaluate correlators in flat space.) So in terms of the usual $n$-point stress tensor correlators, $G_{E}^{\mu \nu, \ldots, \alpha \beta}$ defined in Eq. (2.8) is

$$
\begin{aligned}
G_{E}^{\mu \nu, \alpha \beta}(0, x)= & \left.\left\langle T^{\mu \nu}(0) T^{\alpha \beta}(x)\right\rangle\right|_{g_{\mu \nu}=\eta_{\mu \nu}}+\left.2\left\langle\frac{\partial T^{\mu \nu}(0)}{\partial g_{\alpha \beta}(x)}\right\rangle\right|_{g_{\mu \nu}=\eta_{\mu \nu}} \\
G_{E}^{\mu \nu, \alpha \beta, \gamma \rho}(0, x, y)= & \left.\left\langle T^{\mu \nu}(0) T^{\alpha \beta}(x) T^{\gamma \rho}(y)\right\rangle\right|_{g_{\mu \nu}=\eta_{\mu \nu}}+\left.2\left\langle\frac{\partial T^{\mu \nu}(0)}{\partial g_{\alpha \beta}(x)} T^{\gamma \rho}(y)\right\rangle\right|_{g_{\mu \nu}=\eta_{\mu \nu}} \\
& +\left.2\left\langle\frac{\partial T^{\mu \nu}(0)}{\partial g_{\gamma \rho}(y)} T^{\alpha \beta}(x)\right\rangle\right|_{g_{\mu \nu}=\eta_{\mu \nu}}+\left.2\left\langle T^{\mu \nu}(0) \frac{\partial T^{\alpha \beta}(x)}{\partial g_{\gamma \rho}(y)}\right\rangle\right|_{g_{\mu \nu}=\eta_{\mu \nu}} \\
& +\left.4\left\langle\frac{\partial^{2} T^{\mu \nu}(0)}{\partial g_{\alpha \beta}(x) \partial g_{\gamma \rho}(y)}\right\rangle\right|_{g_{\mu \nu}=\eta_{\mu \nu}} .
\end{aligned}
$$

The terms involving derivatives of the stress tensor are called contact terms, and are discussed in some detail in Ref. [23]. Since $\partial T^{\mu \nu}(x) / \partial g_{\alpha \beta}(y) \propto \delta^{4}(x-y)$ they have very simple momentum dependence. In particular, the contact term in $G_{E}^{\mu \nu, \alpha \beta}(x)$ is $\propto \delta^{4}(x)$; so its contribution to $G_{E}^{\mu \nu, \alpha \beta}(k)$ is $k$-independent. Therefore it does not contribute to Eq. (2.10).

Now consider the four contact terms in Eq. (3.2) and their contribution to Eq. (2.11). Defining

$$
2 \frac{\partial T^{\mu \nu}(x)}{\partial g_{\alpha \beta}(y)} \equiv X^{\mu \nu \alpha \beta} \delta^{4}(x-y)
$$

we find three $X$-type contact terms, involving $\delta^{4}(x), \delta^{4}(y)$, and $\delta^{4}(x-y)$ respectively. The first gives a contribution which is independent of $p$ and so does not contribute to Eq. (2.11); similarly the second is independent of $q$ and also does not contribute. But the third term does contribute;

$$
\begin{aligned}
\lambda_{3}= & -4 \lim _{p_{z}, q_{z} \rightarrow 0} \partial_{p_{z}} \partial_{q_{z}}\left\langle T^{x t}(p) T^{y t}(q) T^{x y}(-p-q)\right\rangle \\
& -4 \lim _{p_{z}, q_{z} \rightarrow 0} \partial_{p_{z}} \partial_{q_{z}}\left\langle X^{x t y t}(p+q) T^{x y}(-p-q)\right\rangle .
\end{aligned}
$$

In order to calculate these transport coefficients for a generic field theory, we need to find the explicit form of both the stress tensor $T^{\mu \nu}$ and of the contact term $X^{\mu \nu \alpha \beta}$ by differentiating the action

$$
S=\int d^{4} x\left(\mathcal{L}_{\text {scalar }}+\mathcal{L}_{\text {spinor }}+\mathcal{L}_{\text {vector }}\right)
$$


with respect to the metric. Since we only attempt a leading-order calculation here, it is sufficient to consider the free-theory action in curved space,

$$
\begin{aligned}
\mathcal{L}_{\text {scalar }} & =\frac{\sqrt{g}}{2} g^{\mu \nu} \partial_{\nu} \phi \partial_{\mu} \phi \\
\mathcal{L}_{\text {spinor }} & =|e| \bar{\psi} \gamma^{c} e_{c}^{\lambda}\left(\partial_{\lambda}+\frac{1}{2} G^{a b} \omega_{\lambda}^{a b}\right) \psi, \\
\mathcal{L}_{\text {vector }} & =\frac{\sqrt{g}}{4} F_{\mu \nu} F_{\rho \tau} g^{\mu \rho} g^{\nu \tau} .
\end{aligned}
$$

Here $g^{\mu \nu}$ is the inverse of $g_{\mu \nu}, F_{\mu \nu}=\partial_{\mu} A_{\nu}-\partial_{\nu} A_{\mu}$ is the field strength tensor, $e_{\mu}^{a}$ is the vierbein related to $g_{\mu \nu}$ by $\eta_{a b} e_{\mu}^{a} e_{\nu}^{b}=g_{\mu \nu}$ and $|e|=\operatorname{det}\left(e_{\mu}^{a}\right)$ is its determinant. Finally, $\omega_{\lambda}^{a b}$ is the spin connection and $G^{a b}=\frac{1}{4}\left[\gamma^{a}, \gamma^{b}\right]$.

Actually, more generally the scalar Lagrangian density should read

$$
\mathcal{L}_{\text {scalar }}=\frac{\sqrt{g}}{2}\left(g^{\mu \nu} \partial_{\nu} \phi \partial_{\mu} \phi-\xi R \phi^{2}\right)
$$

where $\xi$ is a dimensionless constant and $R$ is the Ricci scalar introduced earlier. The action is conformal for the choice $\xi=\frac{1}{6}$ and is called minimally coupled if $\xi=0$ [24]. We will consider general $\xi$, but in the end our results for $\kappa, \lambda_{3}$ are $\xi$ independent.

Through the reminder of this section we will compute $\kappa$ and $\lambda_{3}$ using Eq. (2.10) and Eq. (3.4), applying the action in Eq. (3.5). We also note that at the leading order calculation, $\lambda_{4}=0$ due to conformal symmetry. Since the effect of more degrees of freedom $N_{0}, N_{1 / 2}$ and $N_{1}$ at this level is multiplicative, they can be counted in the final result respectively.

\subsection{Scalars}

In carrying out the variation of Eq. (3.9) with respect to $g_{\mu \nu}$, we must consider the explicit dependence and the implicit dependence via the Ricci scalar $R$. The resulting stress tensor is

$$
T^{\mu \nu}=\left((1-2 \xi) g^{\mu \alpha} g^{\nu \beta}+\frac{4 \xi-1}{2} g^{\mu \nu} g^{\alpha \beta}\right) \partial_{\alpha} \phi \partial_{\beta} \phi+2 \xi\left(g^{\mu \nu} g^{\alpha \beta}-g^{\mu \alpha} g^{\nu \beta}\right) \phi \partial_{\alpha} \partial_{\beta} \phi,
$$

plus terms which vanish in flat space.

With $T^{\mu \nu}$ in hand, we can compute the scalar contribution to $\kappa$. The lowest order diagram is shown in Figure 1 . The momentum $k$ enters at one $T^{x y}$ insertion and exits at the other, so the scalar propagators carry different momenta, $p$ and $p+k \equiv q . T^{x y}$ inserted between these lines obeys the Feynman rule (note directions of momentum flow)

$$
\underset{p \rightarrow}{T^{x y}} \frac{}{q \rightarrow}=(1-2 \xi)\left(p^{x} q^{y}+q^{x} p^{y}\right)+2 \xi\left(p^{x} p^{y}+q^{x} q^{y}\right)
$$




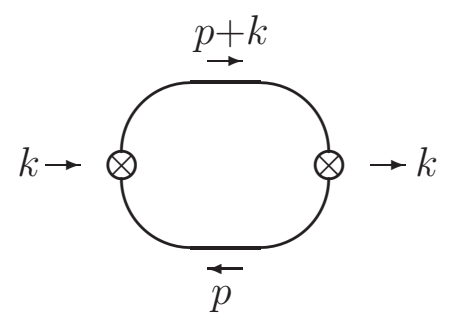

Figure 1. Leading order scalar diagram contributing to $\left\langle T^{x y}(-k) T^{x y}(k)\right\rangle$, necessary for evaluation of $\kappa$. The crosses are $T$ insertions, the solid lines are scalar propagators, and the arrows indicate the momenta flowing on lines and entering or leaving $T$ insertions.

Since we are only differentiating with respect to $k_{z}$, we may set $k_{x}=0=k_{y}$ from the outset, in which case $p^{x}=q^{x}$ and $p^{y}=q^{y}$. Therefore the $\xi$ terms cancel ${ }^{4}$ and the diagram evaluates to

$$
\begin{aligned}
\kappa=\partial_{k_{z}}^{2}\left\langle T^{x y}(-k) T^{x y}(k)\right\rangle & =\left.\frac{1}{2} \partial_{k_{z}}^{2} \oint_{p} \frac{\left(2 p^{x} p^{y}\right)^{2}}{p^{2}(p+k)^{2}}\right|_{k=0} \\
& =-4 T \sum_{p}\left(\frac{1}{p^{6}}-\frac{4 p_{z}^{2}}{p^{8}}\right) p_{x}^{2} p_{y}^{2}=-\frac{T^{2}}{72},
\end{aligned}
$$

where $\frac{1}{2}$ is the symmetry factor of the diagram, and the integration-summation symbol is defined as

$$
\sum_{p}=T \sum_{p^{0}=2 \pi n T} \int \frac{d^{3} \vec{p}}{(2 \pi)^{3}}
$$

and $n$ runs over the integers. In evaluating this and related sum-integrals we use the result

$$
\sum_{p} \frac{\left(\vec{p}^{2}\right)^{n}}{\left(p^{2}\right)^{n+1}}=\frac{(2 n+1) !}{2^{2 n}(n !)^{2}} \frac{T^{2}}{12} .
$$

Expressions with powers of $\left(p^{0}\right)^{2}$ in the numerator can be handled by rewriting $\left(p^{0}\right)^{2}=p^{2}-\vec{p}^{2}$ and using this relation repeatedly; for instance, $\mathbb{S}_{p} \frac{\left(p^{0}\right)^{6}}{\left(p^{2}\right)^{4}}=\frac{-1}{16} \frac{T^{2}}{12}$. We handle $p_{x}^{2} p_{y}^{2} p_{z}^{2}$ by angular averaging, $\left\langle p_{x}^{2} p_{y}^{2} p_{z}^{2}\right\rangle_{\text {angle }}=\vec{p}^{6} / 105$.

Our leading-order result for $\kappa$ agrees with the result in Ref. [23]. The above result shows that the weak coupling expansion of $\kappa$ starts at $\alpha^{0}$.

Now we turn to the computation of $\lambda_{3}$. The first term appearing in Eq. (3.4) is represented by the diagram shown in Figure 2. Once again, $p, q$ only need have nonvanishing $z$-components, but the $T$ operators only return $x, y, 0$ components, which simplifies the evaluation of the diagram and ensures that the result is $\xi$ independent.

\footnotetext{
${ }^{4}$ The same will not happen if we compute non-conformal coefficients.
} 


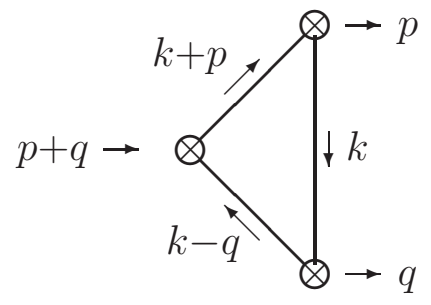

Figure 2. Three-point correlation function $\left\langle T^{x t}(p) T^{y t}(q) T^{x y}(-p-q)\right\rangle$ that contributes to the Kubo formula of $\lambda_{3}$; the leftmost vertex is $T^{x y}$, the other vertices are $T^{x t}$ and $T^{y t}$.

The diagram evaluates to

$$
\begin{aligned}
& -4 \partial_{p_{z}} \partial_{q_{z}}\left\langle T^{x t}(p) T^{y t}(q) T^{x y}(-p-q)\right\rangle \\
= & -\left.4 \partial_{p_{z}} \partial_{q_{z}} \oint_{k} \frac{\left(2 k^{x} k^{y}\right)\left(2 k^{t} k^{x}\right)\left(2 k^{t} k^{y}\right)}{(k+p)^{2}(k-q)^{2} k^{2}}\right|_{p, q=0} \\
= & 128 \oint_{k} \frac{k_{t}^{2} k_{x}^{2} k_{y}^{2} k_{z}^{2}}{k^{10}}=-\frac{T^{2}}{36} .
\end{aligned}
$$

For the contact term in Eq. (3.4), we need to calculate $X^{y t x t}$ as defined in Eq. (3.3). Variation of Eq. (3.10) with respect to $g_{x t}$ gives gives

$$
X^{y t x t}=-\partial^{y} \phi \partial^{x} \phi,
$$

in flat space and for $\xi=0$. In addition there are terms proportional to $\xi$, but they again always involve the combination $\partial_{\alpha} \partial_{\beta} \phi^{2}$. These terms do not contribute to the correlation function we need for the same reason the $\xi$-proportional terms above did not contribute; the incoming and outgoing momenta equal for the components which make up the indices of $X^{t x t y}$. Therefore the result is again $\xi$ independent. The contribution from the $\langle T X\rangle$ correlator to $\lambda_{3}$ is

$$
\begin{aligned}
-4 \partial_{p_{z}} \partial_{q_{z}}\left\langle X^{x t y t}(p+q) T^{x y}(-p-q)\right\rangle & =2 \partial_{p_{z}} \partial_{q_{z}} \oint_{k} \frac{\left(2 k_{x} k_{y}\right)^{2}}{(k-p)^{2}(k+q)^{2}} \\
& =-32 \sum_{k} \frac{k_{x}^{2} k_{y}^{2} k_{z}^{2}}{\left(k^{2}\right)^{4}}=-\frac{T^{2}}{18} .
\end{aligned}
$$

This diagram is actually the same as the diagram which determines $\kappa$; shifting the integration variable used above by $p$, the integral becomes the same one needed in evaluating $\kappa$ except for the overall factor of 4. Summing up Eq. (3.15) and Eq. (3.17), we get

$$
\lambda_{3}=-\frac{T^{2}}{12} \quad 1 \text { real scalar field }
$$

We follow a similar calculation for gauge and fermion fields in the next sections. 


\subsection{Gauge fields}

The gauge field stress tensor derived by $g_{\mu \nu}$ variation using the gauge field action, Eq. (3.8), is

$$
T^{\mu \nu}=F^{\mu \alpha} F_{\alpha}^{\nu}-\frac{1}{4} g^{\mu \nu} F^{\alpha \beta} F_{\alpha \beta},
$$

and from the above relation, we derive the Feynman rule for the vertex,

$$
\begin{aligned}
& \mu \underset{p \rightarrow \operatorname{Tos}_{k \rightarrow}^{\alpha \beta} \sim \nu, b}{\sim} \delta_{a b}\left(\left(p_{\alpha} g_{\mu \gamma}-p_{\gamma} g_{\mu \alpha}\right)\left(k_{\beta} g_{\nu}^{\gamma}-k^{\gamma} g_{\nu \beta}\right)+(\mu \leftrightarrow \nu)\right. \\
& \left.-g_{\alpha \beta}\left(p \cdot k g_{\mu \nu}-k_{\mu} p_{\nu}\right)\right) \text {. }
\end{aligned}
$$

The expression for $X^{\mu \nu \alpha \beta}$ is rather long, but for the case of interest, $X^{t x t y}$, it is quite simple:

$$
X^{t x t y}=-F^{x z} F^{y}
$$

leading to the Feynman rule

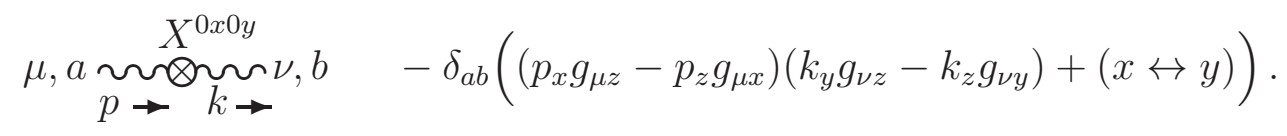

The calculation of $\kappa$ and $\lambda_{3}$ then proceeds via the same diagrams as in the scalar case, but with these somewhat more complicated Feynman rules for the vertices, and with gauge propagators. Note that, because $T^{\mu \nu}$ is built from field strengths, it applies a transverse projector onto the incoming gauge field index; contracting Eq. (3.20) with $p_{\mu}$ or $k_{\nu}$ gives zero. Therefore the result is gauge parameter independent within covariant gauges (and all linear gauges). After significant algebra we find that

$$
\kappa=\frac{T^{2}}{18} \text { for a single color }
$$

while the two diagrams contributing to $\lambda_{3}$ give

$$
-4 \partial_{p_{z}} \partial_{q_{z}}\left\langle T^{x t}(p) T^{y t}(q) T^{x y}(-p-q)\right\rangle=\frac{2 T^{2}}{9}
$$

and

$$
-4 \partial_{p_{z}} \partial_{q_{z}}\left\langle X^{x t y t}(p+q) T^{x y}(-p-q)\right\rangle=\frac{T^{2}}{9} .
$$

Therefore, the gauge field contribution to $\lambda_{3}$ is

$$
\lambda_{3}=\frac{T^{2}}{3} \text { for a single color }
$$

Since each color possesses two spin states, we need to divide these results by 2 to get expressions per degree of freedom. 


\subsection{Fermions}

The treatment of fermions in curved space requires the introduction of the vierbein (also called the frame vector or tetrad) $e_{\mu}^{a}$ (for a review and a treatment of their application to the stress tensor see Ref. [25]). The vierbein relates a local orthonormal coordinate system on the tangent space, with indices $a$ and metric $\eta_{a b}$ (which is $\delta_{a b}$ in Euclidean space) to the metric, via

$$
\eta_{a b} e_{\mu}^{a} e_{\nu}^{b}=g_{\mu \nu}
$$

in a sense it is the square root of the metric. The Dirac action is $e_{a}^{\mu} \bar{\psi} \gamma^{a} \nabla_{\mu} \psi$, where the action of $\nabla_{\mu}$ on a spinorial object is determined by the spin connection $\omega_{\mu}^{a b}$ :

$$
\nabla_{\mu} \psi=\partial_{\mu} \psi+\frac{1}{2} G_{[a b]} \omega_{\mu}^{a b} \psi
$$

where $G_{[a b]}=\frac{1}{4}\left[\gamma_{a}, \gamma_{b}\right]$, and the spin connection is related to the vierbein via

$$
\omega_{\mu}^{a b}=\frac{1}{2} e^{a \nu}\left(\partial_{\mu} e_{\nu}^{b}-\partial_{\nu} e_{\mu}^{b}\right)-\frac{1}{2} e^{b \nu}\left(\partial_{\mu} e_{\nu}^{a}-\partial_{\nu} e_{\mu}^{a}\right)+\frac{1}{2} e^{a \nu} e^{b \sigma}\left(\partial_{\sigma} e_{\nu}^{c}-\partial_{\nu} e_{\sigma}^{c}\right) e_{c \mu} .
$$

Because the action depends on the local frame components, the stress-tensor for fermions cannot be obtained by functional differentiation with respect to the metric tensor; instead one must use the more general expression $T^{\mu \nu}(x)=e_{a}^{\nu} \frac{\partial Z}{\partial e_{a \mu}(x)}$, which reduces to $T^{\mu \nu}(x)=2 \frac{\partial Z}{\partial g_{\mu \nu}(x)}$ for any terms which depend only on $g_{\mu \nu}$ because of Eq. (3.27). Applying this relation to the fermionic action, noting that $\frac{\delta e^{a \mu}}{\partial e_{b \nu}}=-\eta^{a b} g^{\mu \nu}$ (since $g^{\mu \nu}$ is the inverse of $g_{\mu \nu}$; alternatively, because the variation of $g^{\mu \nu} g_{\mu \nu}$ should vanish), and specializing to the non-diagonal entries in $T^{\mu \nu}$, after some work one obtains [25]

$$
T^{\mu \nu}=\frac{1}{4}\left(\bar{\psi} \gamma^{\mu} \nabla^{\nu} \psi-\nabla^{\mu} \bar{\psi} \gamma^{\nu} \psi+\bar{\psi} \gamma^{\nu} \nabla^{\mu} \psi-\nabla^{\nu} \bar{\psi} \gamma^{\mu} \psi\right)
$$

The relevant Feynman rule is

$$
\underset{p \rightarrow}{\stackrel{T^{x y}}{\otimes} \underset{q \rightarrow}{\longrightarrow}} \quad \frac{i}{4}\left(\gamma^{x}\left(p^{y}+q^{y}\right)+\gamma^{y}\left(p^{x}+q^{x}\right)\right)
$$

leading to an expression for $\kappa$,

$$
\kappa=-\partial_{k_{z}}^{2} \sum_{p}^{\prime} i^{2}(-i)^{2} \frac{\operatorname{Tr}\left(\left[(2 p+k)^{x} \gamma^{y}+(2 p+k)^{y} \gamma^{x}\right] \not p\left[(2 p+k)^{x} \gamma^{y}+(2 p+k)^{y} \gamma^{x}\right][\not p+\not k]\right)}{16 p^{2}(p+k)^{2}}
$$

where prime on the sum-integral indicates that the frequencies are $(2 n+1) \pi T$. In terms of this fermionic sum-integral, the equivalent of Eq. (3.14) is

$$
\oint_{p}^{\prime} \frac{\left(\vec{p}^{2}\right)^{n}}{\left(p^{2}\right)^{n+1}}=\frac{(2 n+1) !}{2^{2 n}(n !)^{2}} \frac{\left(-T^{2}\right)}{24} \text {. }
$$


The rest of the evaluation is straightforward, yielding

$$
\kappa=\frac{T^{2}}{72} \text { for a single flavor }
$$

Since a Dirac fermion has 4 degrees of freedom, this should be divided by 4 to get the contribution per degree of freedom.

Next we calculate $\lambda_{3}$ from Eq. (3.4). The three point diagram still looks like Figure 2, but with two terms depending on whether the fermion number follows or opposes the indicated momentum flow. A straightforward evaluation yields a contribution to $\lambda_{3}$ of $T^{2} / 24$.

We specialize immediately to the contact term needed in the calculation; the general expression is not simple. The formula for $X^{t x t y}$ is

$$
X^{t x t y}=\frac{1}{4}\left(e_{a}^{t} \frac{\delta T^{t y}}{\delta e_{a x}}+e_{a}^{x} \frac{\delta T^{t y}}{\delta e_{a t}}+e_{a}^{t} \frac{\delta T^{t x}}{\delta e_{a y}}+e_{a}^{y} \frac{\delta T^{t x}}{\delta e_{a t}}\right) .
$$

Using the techniques already introduced, we find after some work that

$$
X^{t x t y}=\frac{-3}{16}\left(\bar{\psi} \gamma^{x} \nabla^{y} \psi+\bar{\psi} \gamma^{y} \nabla^{x} \psi-\nabla^{x} \bar{\psi} \gamma^{y} \psi-\nabla^{y} \bar{\psi} \gamma^{x} \psi\right)=\frac{-3}{4} T^{x y} .
$$

The $\langle T X\rangle$ diagram contributing to $\lambda_{3}$ therefore gives 3 times the contribution we found for the diagram contributing to $\kappa$, that is, $T^{2} / 24$. Adding these two terms, we find

$$
\lambda_{3}=\frac{T^{2}}{12} \text { for a single flavor. }
$$

Once again, we need to divide by 4 to get the contribution per degree of freedom.

\subsection{Results}

Since we work, so far, at the free theory level, the result is a function only of the number of scalar, spinor, and vector degrees of freedom, which we will write as $N_{0}$, $N_{\frac{1}{2}}, N_{1} \cdot{ }^{5}$ Combining the results of the previous subsections, we find

$$
\begin{aligned}
\kappa & =\frac{T^{2}}{288}\left(-4 N_{0}+N_{\frac{1}{2}}+8 N_{1}\right)+\mathcal{O}(\sqrt{\alpha}), \\
\lambda_{3} & =\frac{T^{2}}{48}\left(-4 N_{0}+N_{\frac{1}{2}}+8 N_{1}\right)+\mathcal{O}(\sqrt{\alpha}) .
\end{aligned}
$$

The other coefficients vanish because the theory is conformal at this order. Curiously, at the free level $\lambda_{3}=6 \kappa$ regardless of the matter content.

We have computed only the leading, coupling-independent contributions. We expect the first corrections to $\kappa, \lambda_{3}$ to arise at $\mathcal{O}\left(\alpha^{\frac{1}{2}}\right)$, and the first contributions to the nonconformal coefficients to be $\mathcal{O}(\alpha)$.

\footnotetext{
${ }^{5} N_{0}$ is 1 per real scalar field; $N_{\frac{1}{2}}$ is two per Weyl spinor field, or 4 per Dirac field; and $N_{1}$ is two per massless spin-1 field (one per spin state). For 3-flavor QCD, $N_{0}=0, N_{\frac{1}{2}}=4 \times 3 \times 3=36[4$ for a Dirac spinor, times three colors times three flavors], and $N_{1}=16$ [2 spin states times 8 color combinations]. For $\mathrm{U}(N) \mathcal{N}=4 \mathrm{SYM}$ theory, $N_{0}=6 N^{2}, N_{\frac{1}{2}}=8 N^{2}$, and $N_{1}=2 N^{2}$.
} 


\section{Lattice implementation}

Here we will briefly discuss some of the challenges associated with evaluating the second-order coefficients on the lattice. One challenge we foresee is choosing and correctly normalizing the operators to use on the lattice. Another challenge is dealing with (incorrect or divergent) short-distance behavior of the correlators. We will not discuss the issue of overcoming fluctuations to achieve good statistics; instead we hope that existing techniques [26] will prove sufficient.

In general, an operator written in terms of lattice variables will not correspond to the continuum operator of interest, but will renormalize and mix with all operators with the same symmetry properties. For instance, a proposed lattice implementation of $T^{x y}$ will generically be expressed in terms of the true $T^{x y}$ as

$$
T_{\text {latt }}^{x y}=Z_{T} T_{\text {contin }}^{x y}+\sum_{n} c_{n} \mathcal{O}_{n}^{x y}
$$

where $\mathcal{O}_{n}^{x y}$ are all other operators with the same symmetries as $T^{x y}$ under the lattice symmetry group, and $Z_{T}, c_{n}$ are some coefficients. Generally the operators $\mathcal{O}_{n}$ are higher dimension than $T^{x y}$ and so the $c_{n}$ will carry positive powers of the lattice spacing. Therefore, to the extent that we can take the continuum limit the $\mathcal{O}_{n}$ should be harmless except that they can introduce short-range contributions to correlators. However, both the operation of vacuum subtraction and the small momentum limits associated with any lattice implementation of $\left.\partial_{k_{z}}^{2} G(k)\right|_{k_{z} \rightarrow 0}$ tend to remove sensitivity to short distance contributions to the correlators, so we expect this issue to be under control. ${ }^{6}$ The problem is the renormalization constant $Z_{T}$, which in general must be determined nonperturbatively.

To evaluate $Z_{T}$ it is useful to recall the physical interpretation of the stress tensor. If we make a small change to the geometry, changing $g_{\mu \nu}=\eta_{\mu \nu}$ to $g_{\mu \nu}=\eta_{\mu \nu}+h_{\mu \nu}$, the action changes from

$$
S_{g=\eta}=\int d^{4} x \mathcal{L}_{0} \quad \text { to } \quad S_{g=\eta+h}=\int d^{4} x\left(\mathcal{L}_{0}-\frac{1}{2} h_{\mu \nu} T^{\mu \nu}+\mathcal{O}\left(h^{2}\right)\right),
$$

where $\mathcal{L}_{0}$ is the Lagrangian density evaluated assuming $h_{\mu \nu}=0$. For instance, if we modify the lattice action such that the lattice spacing in the $x$-direction increases, the change in the action, to leading order, is $-\frac{1}{2} h_{x x} T^{x x}$. Similarly, if $-h_{x y} T^{x y}$ is added to the action, the geometry becomes skewed such that the separation between the point $(0,0,0,0)$ and the point $(0, x, y, 0)$ is no longer $\sqrt{x^{2}+y^{2}}$ but is $\sqrt{x^{2}+2 x y h_{x y}+y^{2}}$.

\footnotetext{
${ }^{6}$ The short distance behavior of the stress tensor two-point function is $\left\langle T^{x y}(x) T^{x y}(0)\right\rangle \sim x^{-8}$. For a dimension-6 operator $\mathcal{O}_{n}$, the correlator is $\left\langle T^{x y}(x) \mathcal{O}_{n}(0)\right\rangle \sim a^{2} x^{-10}$. The vacuum subtracted value at short distances is $\mathcal{O}\left(T^{4}\right)$ by OPE arguments, see Ref. [27]; hence $\left\langle T^{x y}(x) \mathcal{O}_{n}(0)\right\rangle_{T} \sim$ $a^{2} T^{4} x^{-6}$. The short distance contribution to $\partial_{k}^{2}\left\langle T \mathcal{O}_{n}\right\rangle(k)$ is $\sim \int_{x} x^{2}\left\langle T(x) \mathcal{O}_{n}(0)\right\rangle \sim \int_{x} a^{2} T^{4} x^{-4}$ which is $\mathcal{O}\left(a^{2}\right)$ and at worst $\log \mathrm{UV}$ divergent. Higher dimension contaminants carry more powers of $(a / x)$ and also contribute at order $a^{2}$.
} 
The general strategy for determining the normalization constant $Z_{T}$ on the stress tensor is then to include the proposed stress tensor, with small coefficient $(-c / 2)$, in the action, and to see how much it changes the effective lattice spacing. For instance, for a diagonal component such as $T^{x x}$, one can measure correlation lengths along the $x$-axis and along other lattice axes, or measure the string tension in the $x y$ and $y z$ planes. The change in distance determines $h_{x x}$, and the relation between the proposed $T^{x x}$ and the true one is $-(c / 2) T_{\text {proposed }}^{x x}=\left(-h_{x x} / 2\right) T_{\text {true }}^{x x}$ (unless the change also modifies other axis lengths, in which case the proposed $T^{x x}$ is a mixture of $T^{x x}$, $T^{y y}$ etc).

This technique has been well developed for diagonal components of the stress tensor, see for instance [28-31]. To our knowledge it is not as well developed for the off-diagonal components. Unfortunately, all of the Kubo relations we have found, specifically Eq. (2.10), Eq. (2.11) and Eq. (2.12), involve correlators of off-diagonal components of $T^{\mu \nu}$. But this is easily fixed by performing rotations in our choice of axes. For the case of $\kappa$, we make a $\theta=\frac{\pi}{4}$ rotation in the $(x, y)$ - plane, which transforms Eq. (2.10) to

$$
\kappa=\frac{1}{4} \lim _{k_{z} \rightarrow 0} \frac{\partial^{2}}{\partial k_{z}^{2}}\left(G_{E}^{x x, x x}(k)-2 G_{E}^{x x, y y}(k)+G_{E}^{y y, y y}(k)\right) .
$$

Of course $G_{E}^{x x, x x}=G_{E}^{y y, y y}$ at vanishing $k_{x, y}$ by lattice symmetries, so only one needs to be evaluated.

The correlation function found in [22] involved all off-diagonal stress tensors. Arnold et al found an expression involving only $T^{y t}(z), T^{x t}(z)$ [32], and we extend it to the nonconformal case in the Appendix, see Eq. (A.35), which we reproduce here:

$$
\lambda_{3}=2 \kappa^{*}-2 \lim _{p^{z}, q^{z} \rightarrow 0} \partial_{p^{z}} \partial_{q^{z}} G_{E}^{y y, t x, t x}(p, q) .
$$

Our expression for $\lambda_{3}$ still involves the non-diagonal stress tensor $T^{x t}$. To reexpress it in terms of diagonal terms we must perform a $45^{\circ}$ rotation between the $x$ and time axes. This requires a change in the implementation of the periodic boundary conditions in Euclidean space, as illustrated in Figure 4. As the figure shows, we typically consider a lattice with principal domain running from $t=0$ to $t=\beta$; equivalently we can say that we consider the field theory over the whole $x, t$ plane, but with an identification map which equates every point $(x, t)$ with the point $(x, t+\beta)$. Introducing rotated coordinates $x^{\prime}=(x+t) / \sqrt{2}$ and $t^{\prime}=(t-x) / \sqrt{2}$, the identification map equates a point at $\left(x^{\prime}, t^{\prime}\right)$ with a point at $\left(x^{\prime}+\beta / \sqrt{2}, t^{\prime}+\beta / \sqrt{2}\right)$. We then choose to work on a lattice grid with principal axes along the $x^{\prime}$ and $t^{\prime}$ directions. We have to pick a principal domain, that is, a region of the plane holding exactly one copy of each equivalence class of points under the periodic identification. One choice is to stick with the band of points with $t \in[0, \beta)$; as illustrated in the figure, the points labeled 1 are identified, as are the points labeled 2, 3 etc. This 

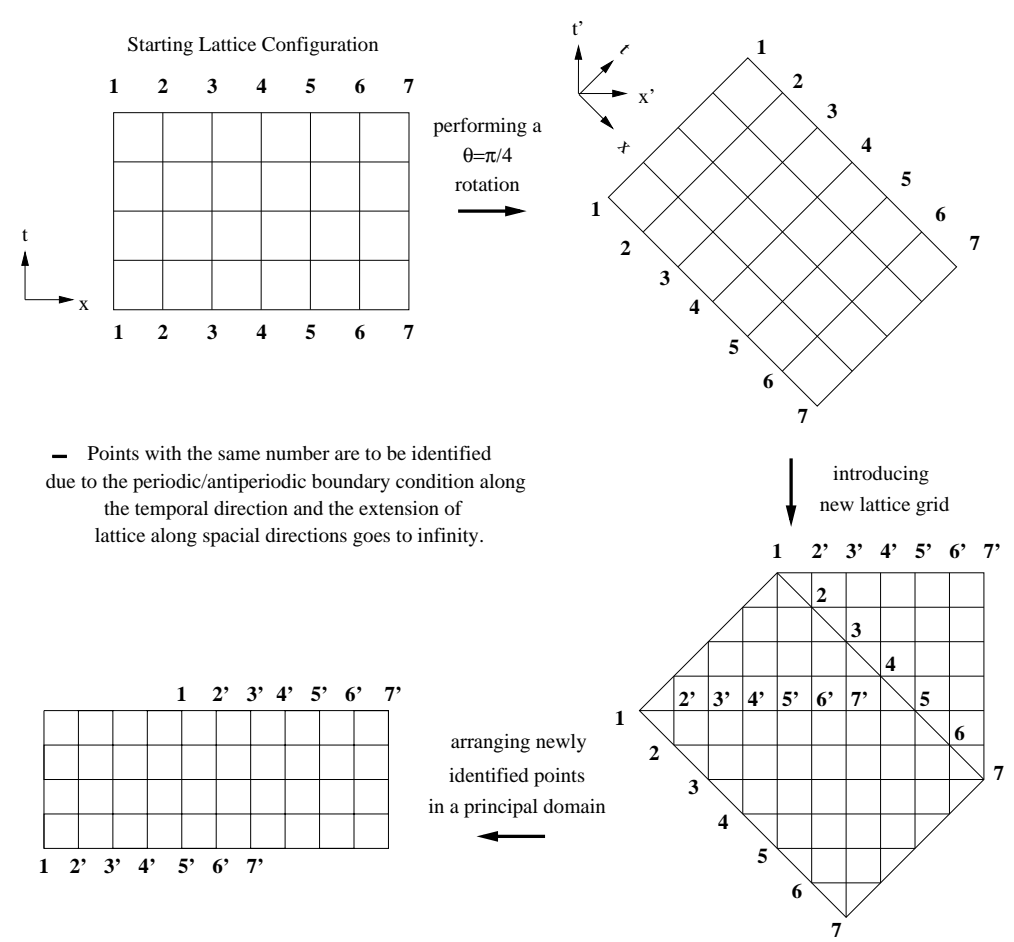

Figure 3. How to handle a rotation which mixes a time and a space direction, on the lattice.

choice is to consider these points as the boundary points on the lattice, which are identified with each other. But the points labeled $2^{\prime}$ are also identified, as are the points labeled $3^{\prime}$ etc. Another choice, as illustrated in the figure, is to choose the band with $t^{\prime} \in[0, \beta / \sqrt{2})$, which has these points as the periodically identified boundary. The identification map relates a point on the bottom edge of this band with a point on the top edge, but shifted over a distance $\beta / \sqrt{2}$ in the $x^{\prime}$ direction. Note that this band is also narrower than the original band; the inverse temperature $\beta$ corresponds to the space separation of the identified points, not the extent of the new "time" coordinate $t^{\prime}$.

Therefore, implementing lattice gauge theory on a space where the periodic identification has a spatial shift corresponds to choosing axes which lie at an angle with respect to the $(x, t)$ axes. In this way we can perform the required $(x, t)$ rotation to make the stress tensor operators needed in the evaluation of $\lambda_{3}$ correspond to diagonal components of the stress tensor. Specifically, in terms of the $x^{\prime}, t^{\prime}$ coordinates after this final rotation, $\lambda_{3}$ is determined by

$$
\lambda_{3}=2 \kappa^{*}-\frac{1}{2} \lim _{p_{z}, q_{z} \rightarrow 0} \frac{\partial^{2}}{\partial p_{z} \partial q_{z}}\left(G_{E}^{t t, t t, y y}(p, q)-2 G_{E}^{t t, x x, y y}(p, q)+G_{E}^{x x, x x, y y}(p, q)\right) .
$$


In a completely analogous way, we find that

$$
\lambda_{4}=\kappa-2 \kappa^{*}-\frac{c_{s}^{4}}{4} \lim _{p, q \rightarrow 0} \frac{\partial^{2}}{\partial p_{x} \partial q_{x}}\left(G_{E}^{t t, t t, x x}(p, q)-G_{E}^{t t, t t, y y}(p, q)\right) .
$$

\section{Discussion}

Our central results are presented in Eq. (3.38) and Eq. (3.39). The thermodynamic coefficients, unlike the entropy-generating coefficients $\eta$ etc, do not diverge in the weak coupling limit, but remain finite. Both $\kappa$ and $\lambda_{3}$ are in general nonzero. In particular, the previous observation that $\lambda_{3}$ vanishes in strongly-coupled $\mathcal{N}=4$ SuperYang-Mills theory $[14,35]$ appears to be an accident.

Curiously, our results for $\kappa$ and $\lambda_{3}$, Eq. (3.38) and Eq. (3.39), yield zero when we insert the matter content of $\mathcal{N}=4$ Super-Yang-Mills theory: $N_{0}=3 N_{1}$ and $N_{\frac{1}{2}}=4 N_{1}$. Therefore, both coefficients vanish in the weak-coupling limit. As we just mentioned, $\lambda_{3}$ also vanishes in this theory in the strong coupling limit. The fact that $\lambda_{3}$ vanishes in both limits is suggestive that it is strictly zero, but this is not the case; it has been shown [36] that $\lambda_{3}$ is nonzero at subleading order in the large-coupling expansion. And it is easy to find other examples of conformal theories where $\lambda_{3}$ is nonzero. For instance, $\mathrm{SU}\left(N_{c}\right)$ gauge theories with fundamental vectorlike matter and with the number of flavors $N_{f}$ slightly below $\frac{11}{2} N_{c}$ have weakly coupled, conformal fixed points [37]. Since such theories are weakly coupled, Eq. (3.39) applies. Both terms are of the same sign, so $\lambda_{3}$ is certainly not zero for these conformal gauge theories.

\section{Acknowledgments}

We thank Simon Caron-Huot and Harvey Meyer for illuminating discussions. This work was supported in part by the Natural Sciences and Engineering Research Council of Canada.

\section{A Non-conformal hydrodynamics}

In this section we derive Kubo formulae for all the second-order, thermodynamical transport coefficients of all non-conformal hydrodynamics. We work in Minkowski space and analytically continue to Euclidean space. Consider a hydrodynamic system in equilibrium with some arbitrary background of form

$$
d s^{2}=\delta_{\mu \nu} d x^{\mu} d x^{\nu}+h_{00}(\vec{x}) d t^{2}+h_{0 i}(\vec{x}) d t d x^{i}+h_{i j}(\vec{x}) d x^{i} d x^{j} .
$$

In this curved background the expectation value of the stress tensor can be expanded about flat space in powers of $h_{\mu \nu}$ :

$$
\left\langle T_{E}^{\mu \nu}\right\rangle_{h}=G_{E}^{\mu \nu}+\int d^{4} x G_{E}^{\alpha \beta, \mu \nu}(x, 0) \frac{h_{\alpha \beta}(x)}{2}+\int d^{4} x d^{4} y G_{E}^{\alpha \beta, \gamma \delta, \mu \nu}(x, y, 0) \frac{h_{\alpha \beta}(x) h_{\gamma \delta}(y)}{8}
$$


where the Euclidean Green functions are defined in Eq. (2.8). The corresponding Minkowski space expression is

$$
\left\langle T_{r}^{\mu \nu}\right\rangle_{h}=G_{r}^{\mu \nu}-\int d^{4} x G_{a r}^{\alpha \beta, \mu \nu}(x, 0) \frac{h_{\alpha \beta}(x)}{2}+\int d^{4} x d^{4} y G_{a a r}^{\alpha \beta, \gamma \delta, \mu \nu}(x, y, 0) \frac{h_{\alpha \beta}(x) h_{\gamma \delta}(y)}{8},
$$

where here the retarded Green functions are the correlation functions of a $T_{r}$ with one or two $T_{a}$, as is explained in detail in [22]. We can shift from each of these two signatures to the other accordingly by noticing that $t$ indices of the Green function are multiplied by a factor of $i$ and also for each $a$ index we get an extra minus sign.

The following expressions for the curvature tensors will be quite handy for the derivations of Kubo formulae. To first and second order of perturbations in the metric of the form, $g_{\mu \nu}(x)=\eta_{\mu \nu}+h_{\mu \nu}(x)$, we have (borrowing the result from [38])

$$
\begin{aligned}
R_{\alpha \beta \gamma \delta}= & S_{\sigma[\delta, \beta \gamma]}-h_{\sigma,[\gamma}^{\lambda} S_{\lambda \delta], \beta}+\frac{1}{2} \eta^{\mu \rho} S_{\sigma[\gamma, \mu} S_{\rho \delta], \beta}+\mathcal{O}\left(h_{\mu \nu}^{3}\right) \\
R_{\beta \delta}= & \frac{1}{2} \eta^{\mu \nu}\left(h_{\mu \delta, \beta \nu}-h_{\beta \delta, \mu \nu}-h_{\mu \nu, \beta \delta}+h_{\beta \nu, \mu \delta}\right)-\left(h_{, \mu}^{\mu \nu}-\frac{1}{2} h^{, \nu}\right) h_{\nu(\delta, \beta)} \\
& +\frac{1}{2}\left(h^{\mu \nu} h_{\beta \delta, \nu}\right)_{, \mu}+\frac{1}{4} h_{, \delta}^{\mu \nu} h_{\mu \nu, \beta}-\frac{1}{4} h^{, \mu} h_{\beta \delta, \mu}+h_{\mu \delta, \nu} h_{\beta}^{[\mu, \nu]}+\frac{1}{2} h^{\mu \nu} h_{\mu \nu, \beta \delta} \\
& -h^{\mu \nu} h_{\mu(\beta, \delta) \nu}+\mathcal{O}\left(h_{\mu \nu}^{3}\right) \\
R= & h_{, \alpha \beta}^{\alpha \beta}-h_{, \alpha}^{\alpha}-h_{, \mu}^{\mu \nu} h_{\nu \alpha}^{, \alpha}+h_{, \mu}^{\mu \nu} h_{, \nu}+\frac{3}{4} h^{\mu \nu, \alpha} h_{\mu \nu, \alpha}-\frac{1}{4} h^{, \mu} h_{, \mu} \\
& -\frac{1}{2} h^{\mu \nu, \alpha} h_{\alpha \mu, \nu}-2 h^{\mu \nu} h_{\mu, \nu \alpha}^{\alpha}+h^{\mu \nu} h_{, \mu \nu}+h^{\mu \nu} h_{\mu \nu, \alpha}{ }^{\alpha}+\mathcal{O}\left(h_{\mu \nu}^{3}\right)
\end{aligned}
$$

and in the above expressions, we have $S_{\lambda \delta, \beta}=h_{\lambda \delta, \beta}+h_{\beta \lambda, \delta}-h_{\beta \delta, \lambda}$.

We will write thermodynamic variables $u^{\mu}, \epsilon, P$ in an expansion about $h=0$; $u^{\mu}(x)=\bar{u}^{\mu}(x)+u_{h}^{\mu}(x)+u_{h^{2}}^{\mu}(x)$ and similarly for $\epsilon, P$ (the barred variables are the $h=0$ values). It is important to understand the role of the rest frame; $u^{\mu}$ need not equal $\bar{u}^{\mu}=(1,0,0,0)$; rather we must determine $u_{h}^{\mu}$ by solving conservation equations, $\nabla_{\mu} T^{\mu \nu}=0$, consistently and truncating the expansion. Finally the fluid vector in equilibrium must satisfy both $\sigma^{\mu \nu}=0$ and $\nabla \cdot u=0$.

We also find it useful to take the trace of the energy-momentum tensor in the case of non-conformal transport coefficients,

$$
T_{\mu}^{\mu}=P\left(3-\frac{1}{c_{s}^{2}}\right)+3 \Pi .
$$

To find the pressure in terms of the background source, we use equations of motion, in equilibrium we have

$$
\begin{aligned}
0 & =\nabla_{\nu} \nabla_{\mu} T^{\mu \nu} \\
& =\nabla_{\nu} \nabla_{\mu} T_{\text {ideal }}^{\mu \nu}+\nabla_{\nu} \nabla_{\mu} \pi^{\mu \nu}+\nabla_{\nu} \nabla_{\mu}\left(\Delta^{\mu \nu} \Pi\right)
\end{aligned}
$$


where for the ideal fluid, we have

$$
\begin{aligned}
\nabla_{\nu} \nabla_{\mu} T_{\text {ideal }}^{\mu \nu}= & u^{\mu} u^{\nu} \nabla_{\nu} \nabla_{\mu}(\epsilon+P)+(\epsilon+P) \nabla_{\nu} \nabla_{\mu}\left(u^{\mu} u^{\nu}\right) \\
& +\nabla_{\mu}(\epsilon+P) \nabla_{\nu}\left(u^{\mu} u^{\nu}\right)+\nabla_{\nu}(\epsilon+P) \nabla_{\mu}\left(u^{\mu} u^{\nu}\right)+\square P .
\end{aligned}
$$

Since $u_{h}^{i}, u_{h^{2}}^{i} \rightarrow 0$ for $\omega \rightarrow 0$, the first term is identically zero, for the second term we get

$$
\begin{aligned}
(\epsilon+P) \nabla_{\nu} \nabla_{\mu}\left(u^{\mu} u^{\nu}\right) & =(\epsilon+P)\left(\nabla_{\nu} u^{\nu} \nabla_{\mu} u^{\mu}+u^{\nu} \nabla_{\nu} \nabla_{\mu} u^{\mu}+\nabla_{\nu} u^{\mu} \nabla_{\mu} u^{\nu}+u^{\mu} \nabla_{\nu} \nabla_{\mu} u^{\nu}\right) \\
& \simeq(\epsilon+P)\left(\left(\Gamma_{\nu \lambda}^{\nu} u^{\lambda}\right)^{2}+\Gamma_{\nu \alpha}^{\mu} \Gamma_{\mu \beta}^{\nu} u^{\alpha} u^{\beta}+R_{\sigma \mu} u^{\sigma} u^{\mu}\right)+\mathcal{O}\left(\omega, h_{\mu \nu}^{3}\right)
\end{aligned}
$$

and we used $\left[\nabla_{\mu}, \nabla_{\nu}\right] u^{\rho}=R_{\sigma \mu \nu}^{\rho} u^{\sigma}$. Similarly, for the third and fourth term in Eq. (A.7), we get

$$
\nabla_{\mu}(\epsilon+P) \nabla_{\nu}\left(u^{\mu} u^{\nu}\right)+\nabla_{\nu}(\epsilon+P) \nabla_{\mu}\left(u^{\mu} u^{\nu}\right) \simeq 2 \partial_{\alpha}(\epsilon+P) \Gamma_{\beta \gamma}^{\alpha} \bar{u}^{\beta} \bar{u}^{\gamma}+\mathcal{O}\left(\omega, h_{\mu \nu}^{3}\right) .
$$

Adding up previous results, finally the pressure reads

$$
P=\bar{P}-\frac{\nabla_{\mu} \nabla_{\nu} \pi^{\mu \nu}+R_{\sigma \mu} \bar{u}^{\sigma} \bar{u}^{\mu}(\epsilon+P+\Pi)+\chi}{\square}-\Pi+\mathcal{O}\left(\omega, h_{\mu \nu}^{3}\right)
$$

where $\square=\sum_{i=1}^{3} \partial_{i}^{2}$ and

$$
\chi=(\epsilon+P)\left(\left(\Gamma_{\nu \lambda}^{\nu} u^{\lambda}\right)^{2}+\Gamma_{\nu \alpha}^{\mu} \Gamma_{\mu \beta}^{\nu} u^{\alpha} u^{\beta}+R_{\sigma \mu} u^{\sigma} u^{\mu}\right)+2 \partial_{\alpha}(\epsilon+P) \Gamma_{\beta \gamma}^{\alpha} \bar{u}^{\beta} \bar{u}^{\gamma} .
$$

This is the generalization of the result in [32]. As pointed out in the main text, some Kubo formulae don't directly relate a transport coefficient to the zero frequency and momentum limit of Green's functions but they mix these parameters. Throughout the next section we'll try to find the simplest setup that can give rise to Green's functions manageable for lattice calculations.

\section{A.1 Kubo relation for $\kappa$ and $\xi_{5}$}

We will start the calculation with $\xi_{5}$, by turning on an $h_{x y}(x, y)$ perturbation and evaluating $\left\langle T^{t t}\right\rangle$. But this is $\epsilon$, the energy density of the fluid, we can find it at different orders of $h_{\mu \nu}$ from Eq. (A.9) or by solving the equations of motion directly. For illustrative reasons, we do the second approach. Since only $h_{x y}(x, y)$ is nonzero we can assume $u_{h}^{\mu}$ is also a function only of $x, y$. The viscous tensor is

$$
\begin{aligned}
\Pi^{x x} & =\frac{1}{3} \frac{\partial^{2} h_{x y}(x, y)}{\partial x \partial y}\left(\kappa+6 \xi_{5}\right)+\frac{2 \eta}{3}\left(\frac{\partial u_{h}^{y}}{\partial y}-2 \frac{\partial u_{h}^{x}}{\partial x}\right)-\zeta\left(\frac{\partial u_{h}^{y}}{\partial y}+\frac{\partial u_{h}^{x}}{\partial x}\right),(A \\
\Pi^{y y} & =\frac{1}{3} \frac{\partial^{2} h_{x y}(x, y)}{\partial x \partial y}\left(\kappa+6 \xi_{5}\right)+\frac{2 \eta}{3}\left(\frac{\partial u_{h}^{x}}{\partial x}-2 \frac{\partial u_{h}^{y}}{\partial y}\right)-\zeta\left(\frac{\partial u_{h}^{y}}{\partial y}+\frac{\partial u_{h}^{x}}{\partial x}\right) \\
\Pi^{z z} & =\frac{1}{3} \frac{\partial^{2} h_{x y}(x, y)}{\partial x \partial y}\left(-2 \kappa+6 \xi_{5}\right)+\frac{2 \eta}{3}\left(\frac{\partial u_{h}^{x}}{\partial x}+\frac{\partial u_{h}^{y}}{\partial y}\right)-\zeta\left(\frac{\partial u_{h}^{y}}{\partial y}+\frac{\partial u_{h}^{x}}{\partial x}\right), \\
\Pi^{x y} & =-\eta\left(\frac{\partial u_{h}^{y}}{\partial x}+\frac{\partial u_{h}^{x}}{\partial y}\right), \quad \Pi^{x z}=-\eta \frac{\partial u_{h}^{z}}{\partial x}, \quad \Pi^{y z}=-\eta \frac{\partial u_{h}^{z}}{\partial y} \\
\Pi & =-\zeta\left(\frac{\partial u_{h}^{y}}{\partial y}+\frac{\partial u_{h}^{x}}{\partial x}\right)+2 \xi_{5} \frac{\partial^{2} h_{x y}(x, y)}{\partial x \partial y} .
\end{aligned}
$$


here and through the following sections we neglect dissipative terms since they will be proportional to the time derivatives of $h_{\mu \nu}$ or fluid vector in general.

Solving the equations of motion for $\nabla_{\mu} T^{\mu x}=0, \nabla_{\mu} T^{\mu y}=0, \nabla_{\mu} T^{\mu z}=0$, $\nabla_{\mu} T^{\mu t}=0$, we get accordingly,

$$
\begin{aligned}
\frac{\partial P_{h}}{\partial x}+\frac{\partial u_{h}^{x}}{\partial t}(\bar{\epsilon}+\bar{P})+\frac{\partial^{3} h_{x y}}{\partial y \partial x^{2}}\left(\frac{\kappa}{3}+2 \xi_{5}\right)-\zeta\left(\frac{\partial^{2} u_{h}^{y}}{\partial x \partial y}+\frac{\partial^{2} u_{h}^{x}}{\partial x^{2}}\right) & \\
-\eta\left(\frac{4}{3} \frac{\partial^{2} u_{h}^{x}}{\partial x^{2}}+\frac{\partial^{2} u_{h}^{x}}{\partial y^{2}}+\frac{1}{3} \frac{\partial^{2} u_{h}^{y}}{\partial x \partial y}\right) & =0 \\
\frac{\partial P_{h}}{\partial y}+\frac{\partial u_{h}^{y}}{\partial t}(\bar{\epsilon}+\bar{P})+\frac{\partial^{3} h_{x y}}{\partial x \partial y^{2}}\left(\frac{\kappa}{3}+2 \xi_{5}\right)-\zeta\left(\frac{\partial^{2} u_{h}^{x}}{\partial x \partial y}+\frac{\partial^{2} u_{h}^{y}}{\partial y^{2}}\right) & \\
-\eta\left(\frac{4}{3} \frac{\partial^{2} u_{h}^{y}}{\partial y^{2}}+\frac{\partial^{2} u_{h}^{y}}{\partial x^{2}}+\frac{1}{3} \frac{\partial^{2} u_{h}^{x}}{\partial x \partial y}\right) & =0 \\
\frac{\partial u_{h}^{z}}{\partial t}(\bar{\epsilon}+\bar{P})-\eta\left(\frac{\partial^{2} u_{h}^{z}}{\partial x^{2}}+\frac{\partial^{2} u_{h}^{z}}{\partial y^{2}}\right) & =0 \\
\frac{\partial u_{h}^{x}}{\partial x}(\bar{\epsilon}+\bar{P})+\frac{\partial u_{h}^{y}}{\partial y}(\bar{\epsilon}+\bar{P})+\frac{\partial \epsilon_{h}}{\partial t} & =0 .
\end{aligned}
$$

Since we are interested in the zero frequency limit all time derivatives are zero. As we can see in the above equations terms proportional to the metric perturbation appear, which act as a source for $P$ and for $u^{\mu}$. Higher order terms that include the interaction of the fluid vector with background perturbation have been neglected. From the first two equations we obtain

$$
P_{h}=-\left(\frac{\kappa}{3}+2 \xi_{5}\right) \frac{\partial^{2} h_{x y}}{\partial y \partial x}+\mathcal{O}\left(\partial_{t}\right)
$$

and we know that pressure and energy density are related through $P=c_{s}^{2} \epsilon$. Similarly we get

$u_{h}^{x}=\frac{1}{3} \frac{\omega h_{x y} q_{x}^{2} q_{y}\left(6 \xi_{5}+\kappa\right)}{c_{s}^{2}\left(q_{x}^{2}+q_{y}^{2}\right)}(\bar{\epsilon}+\bar{P})+\mathcal{O}\left(\omega^{2}\right), \quad u_{h}^{y}=\frac{1}{3} \frac{\omega h_{x y} q_{y}^{2} q_{x}\left(6 \xi_{5}+\kappa\right)}{c_{s}^{2}\left(q_{x}^{2}+q_{y}^{2}\right)}(\bar{\epsilon}+\bar{P})+\mathcal{O}\left(\omega^{2}\right)$.

Finally, from Eq. (A.3), we have

$$
\frac{-1}{c_{s}^{2}}\left(\frac{\kappa}{3}+2 \xi_{5}\right) \frac{\partial^{2} h_{x y}}{\partial y \partial x}=-\frac{1}{2} \int d^{4} x G_{a r}^{\alpha \beta, t t}(x, 0) h_{\alpha \beta}(x) .
$$

Fourier transforming and taking the variation of both sides with respect to $h_{x y}$, we get

$$
\xi_{5}=-\frac{c_{s}^{2}}{2} \lim _{k_{x}, k_{y} \rightarrow 0} \frac{\partial^{2}}{\partial k_{x} \partial k_{y}} G_{a r}^{x y, t t}(k)-\frac{\kappa}{6}
$$

and we know that

$$
\lim _{k_{0} \rightarrow 0} G_{a r}^{x y, t t}\left(k_{0}, \mathbf{k}\right)=+G_{E}^{x y, t t}\left(k_{0}=0, \mathbf{k}\right)
$$


So finally in Euclidean space we have

$$
\xi_{5}=-\frac{c_{s}^{2}}{2} \lim _{k_{x}, k_{y} \rightarrow 0} \frac{\partial^{2}}{\partial k_{x} \partial k_{y}} G_{E}^{x y, t t}(k)-\frac{\kappa}{6} .
$$

Similarly the Kubo relation for $\kappa$ in terms of off-diagonal components of stresstensors is given by $\kappa=-\partial_{k_{z}}^{2} G_{a r}^{x y, x y}(k)$. In terms of the Euclidean correlator this is $\kappa=\partial_{k_{z}}^{2} G_{E}^{x y, x y}(k)$.

\section{A.2 Kubo relation for $\kappa^{*}$ and $\xi_{6}$}

To find a Kubo relation for $\xi_{6}$, we use the perturbation $h_{t t}(z)$. This choice, shifts the local rest frame by $u^{t}=1+1 / 2 h_{t t}+\mathcal{O}\left(h^{2}\right)$. Expanding the fluid vector in terms of metric perturbation $h_{\mu \nu}$, we find the following viscous tensors,

$$
\begin{aligned}
\Pi^{x x} & =\Pi^{y y}=\frac{1}{6} \frac{\partial^{2} h_{t t}}{\partial z^{2}}\left(-2 \kappa^{*}+\kappa-3 \xi_{6}+6 \xi_{5}\right) \\
\Pi^{z z} & =\frac{1}{6} \frac{\partial^{2} h_{t t}}{\partial z^{2}}\left(4 \kappa^{*}-2 \kappa-3 \xi_{6}+6 \xi_{5}\right) \\
\Pi & =-\frac{\xi_{6}}{2} \frac{\partial^{2} h_{t t}}{\partial z^{2}}+\xi_{5} \frac{\partial^{2} h_{t t}}{\partial z^{2}} .
\end{aligned}
$$

If we assume that the hydrodynamic waves are only functions of $z$ then for $\nabla_{\mu} T^{\mu z}=0$ we have

$$
0=\frac{\partial P_{h}}{\partial z}+\frac{\partial u_{h}^{z}}{\partial t}(\bar{\epsilon}+\bar{P})-\frac{1}{2} \frac{\partial h_{t t}}{\partial z}(\bar{\epsilon}+\bar{P})+\frac{\partial^{3} h_{t t}}{\partial z^{3}}\left(-\frac{\xi_{6}}{2}+\frac{2 \kappa^{*}}{3}-\frac{\kappa}{3}+\xi_{5}\right)
$$

and once again the last term acts as a source for pressure. For $\left\langle T^{t t}\right\rangle$ we have

$$
\left\langle T^{t t}\right\rangle_{h}=\frac{(\bar{\epsilon}+\bar{P})}{2 c_{s}^{2}} h_{t t}(z)+\left(\frac{\xi_{6}}{2}-\frac{2 \kappa^{*}}{3}+\frac{\kappa}{3}-\xi_{5}\right) \frac{\partial_{z}^{2} h_{t t}(z)}{c_{s}^{2}} .
$$

The first term in the above relation is a pure gauge. For the second term from linear-response we have

$$
\left(\frac{\xi_{6}}{2}-\frac{2 \kappa^{*}}{3}+\frac{\kappa}{3}-\xi_{5}\right) \frac{\partial_{z}^{2} h_{t t}(z)}{c_{s}^{2}}=-\frac{1}{2} \int d^{4} x G_{a r}^{\alpha \beta, t t}(x, 0) h_{\alpha \beta}(x) .
$$

The corresponding Kubo formula for $\xi_{6}$ will be

$$
\xi_{6}=2 \xi_{5}+\frac{4 \kappa^{*}}{3}-\frac{2 \kappa}{3}+\frac{c_{s}^{2}}{2} \lim _{k_{z} \rightarrow 0} \frac{\partial^{2}}{\partial k_{z}^{2}} G_{a r}^{t t, t t}(k)
$$

and accordingly in Euclidean space using $\lim _{k_{t} \rightarrow 0} G_{a r}^{t t, t t}\left(k_{t}, \mathbf{k}\right)=-G_{E}^{t t, t t}\left(k_{t}=0, \mathbf{k}\right)$, it can be rewritten as

$$
\xi_{6}=2 \xi_{5}+\frac{4 \kappa^{*}}{3}-\frac{2 \kappa}{3}-\frac{c_{s}^{2}}{2} \lim _{k_{z} \rightarrow 0} \frac{\partial^{2}}{\partial k_{z}^{2}} G_{E}^{t t, t t}(k) .
$$


This formula determines a linear combination of $\xi_{6}$ and $\kappa^{*}$. To get both coefficients separately we need to look for another relation for $\kappa^{*}$. We do so by investigating an off-diagonal component of energy-momentum tensor. We have

$$
\left\langle T^{x y}\right\rangle=(\epsilon+P) u^{x} u^{y}+P g^{x y}+\Pi^{x y} .
$$

Since $\kappa^{*}$ is a coefficient involving the curvature tensor, which first arises at linear order in $h$, we need only work to this order, in which case the first term is zero; and if we choose $h_{x y}$ nonzero then $P g^{x y}$ and $\Delta^{x y} \Pi$ are also zero. Therefore we consider $h_{t t}(x, y)$. Then the only contribution comes from $\pi^{x y}$, after expansion in the orders of $h_{t t}$ and $u^{\mu}(x)=\bar{u}^{\mu}(x)+u_{h}^{\mu}(x)+\mathcal{O}\left(h^{2}\right)$, we find

$$
\pi^{x y}=\frac{\partial^{2} h_{t t}}{\partial x \partial y}\left(\kappa^{*}-\frac{\kappa}{2}\right)
$$

If we use Eq. (A.3) and Fourier transforming, we can write the Kubo formula in Euclidean space as

$$
\kappa^{*}=\frac{\kappa}{2}+\frac{1}{2} \lim _{k_{x}, k_{y} \rightarrow 0} \frac{\partial^{2}}{\partial k_{x} \partial k_{y}} G_{E}^{x y, t t}(k) .
$$

Eq. (A.27) and Eq. (A.18) involve the same Green function, so we find a relation between $\xi_{5}$ and $\kappa^{*}$, specifically

$$
\xi_{5}=-c_{s}^{2} \kappa^{*}+\frac{\kappa}{2}\left(c_{s}^{2}-\frac{1}{3}\right) .
$$

\section{A.3 Kubo relation for $\lambda_{3}$ and $\xi_{3}$}

Now we turn to nonlinear transport coefficients, where we must work to second order in $h$. We begin with $\lambda_{3}$, which is the traceless contribution arising at second order in vorticity. Vorticity is generated by a nonvanishing value of $h_{t i, j}$; specifically the vorticity term for which $\lambda_{3}$ is a parameter (see Eq. (2.7)) is

$$
\Omega_{\lambda}^{\langle i} \Omega^{j\rangle \lambda}=\frac{1}{12}\left(\delta^{i j} \delta_{m n}-3 \delta_{m}^{i} \delta_{n}^{j}\right) \epsilon^{m k l} \epsilon^{n r s} \partial_{k} h_{l t} \partial_{r} h_{s t}
$$

The easiest way to proceed $[22,32]$ is to consider an off-diagonal component of $T$, such as $T^{x y}$; then complications involving the pressure (such as those of the last subsections) do not arise. However as we have discussed it is most convenient on the lattice to use a relation involving a diagonal component of the stress tensor. Therefore we will instead consider the vorticity-related contributions to $T^{x x}$ :

$$
\left\langle T^{x x}\right\rangle=(\epsilon+p) u^{x} u^{x}+p g^{x x}+\Pi^{x x} .
$$

Since we have to keep all terms to the order of $\mathcal{O}\left(h^{2}\right)$, we need to know $u_{h}^{x}, P_{h^{2}}$, and $\Pi_{h^{2}}^{x x}$. We will consider nonvanishing $h_{t y}(z)$, which is general enough for $\Pi^{x x}$ to contain a $\lambda_{3}$ dependent term. For this choice $u_{h}^{x}$ vanishes. To find the contribution 
of $P_{h^{2}}$, we look into Eq. (A.9). Since $\Gamma_{t t}^{\nu}$ is zero for our metric perturbation, the only possible contributions come from $\partial_{\mu} \partial_{\nu} \pi^{\mu \nu}$ and $\Pi$. All derivatives other than $\partial_{z}$ are zero, so finally we have

$$
P_{h^{2}}=-\pi^{z z}-\Pi=-\Pi^{z z} .
$$

Calculating $\Pi^{z z}, \Pi^{x x}$ and recalling that $u_{h}^{\mu}=0$, the final result reads

$$
\begin{aligned}
& \Pi^{z z}=-h_{t y} \frac{\partial^{2} h_{t y}}{\partial z^{2}}\left(2 \xi_{5}+\frac{\kappa}{3}\right)+\left(\frac{\partial h_{0 y}}{\partial z}\right)^{2}\left(-\frac{3 \xi_{5}}{2}+\frac{\lambda_{3}}{12}+\frac{\xi_{6}}{2}-\frac{\kappa^{*}}{6}+\frac{\xi_{3}}{2}\right)+\mathcal{O}\left(h^{3}\right) \\
& \Pi^{x x}=2 h_{t y} \frac{\partial^{2} h_{t y}}{\partial z^{2}}\left(-\xi_{5}+\frac{\kappa}{3}\right)+\left(\frac{\partial h_{t y}}{\partial z}\right)^{2}\left(-\frac{3 \xi_{5}}{2}-\frac{\lambda_{3}}{6}+\frac{\xi_{6}}{2}+\frac{\kappa^{*}}{3}+\frac{\xi_{3}}{2}\right)+\mathcal{O}\left(h^{3}\right) .
\end{aligned}
$$

Now for Eq. (A.30) we can write $\left\langle T^{x x}\right\rangle=P+\Pi^{x x}=\bar{P}-\Pi^{z z}+\Pi^{x x}$, which reads

$$
\left\langle T^{x x}\right\rangle-\bar{P}=h_{t y} \frac{\partial^{2} h_{t y}}{\partial z^{2}} \kappa+\left(\frac{\partial h_{t y}}{\partial z}\right)^{2}\left(-\frac{\lambda_{3}}{4}+\frac{\kappa^{*}}{2}\right)+\mathcal{O}\left(h^{3}\right) .
$$

For the response of the three-point Green's function, we have

$$
h_{t y} \frac{\partial^{2} h_{t y}}{\partial z^{2}} \kappa+\left(\frac{\partial h_{t y}}{\partial z}\right)^{2}\left(-\frac{\lambda_{3}}{4}+\frac{\kappa^{*}}{2}\right)=\frac{1}{8} \int d^{4} x d^{4} y G_{a a r}^{\alpha \beta, \gamma \delta, x x}(x, y, 0) h_{\alpha \beta}(x) h_{\gamma \delta}(y)
$$

which results in the following Kubo formula for $\lambda_{3}$ :

$$
\lambda_{3}=2 \kappa^{*}+2 \lim _{p_{z}, q_{z} \rightarrow 0} \frac{\partial^{2}}{\partial p_{z} \partial q_{z}} G_{a a r}^{t y, t y, x x}(p, q),
$$

and in Euclidean space through $\lim _{p_{0}, q_{0} \rightarrow 0} G_{a a r}^{t y, t y, x x}(p, q)=-G_{E}^{t y, t y, x x}(\mathbf{p}, \mathbf{q})$, we get

$$
\lambda_{3}=2 \kappa^{*}-2 \lim _{p_{z}, q_{z} \rightarrow 0} \frac{\partial^{2}}{\partial p_{z} \partial q_{z}} G_{E}^{t y, t y, x x}(p, q) .
$$

In the main text we use this expression but with $x \leftrightarrow y$.

It's quite easy to find a Kubo relation for $\xi_{3}$ now, since we have a relation for $\lambda_{3}$. From the trace of stress-tensor we have

$$
T_{\mu}^{\mu}=P\left(3-\frac{1}{c_{s}^{2}}\right)+3 \Pi .
$$

Using Eq. (A.31) and the expressions for $\Pi^{z z}$ and $\Pi$ that can be calculated as

$$
\Pi=-2 h_{t y} \frac{\partial^{2} h_{t y}}{\partial z^{2}} \xi_{5}+\frac{1}{2}\left(\frac{\partial h_{t y}}{\partial z}\right)^{2}\left(\xi_{6}-3 \xi_{5}+\xi_{3}\right)+\mathcal{O}\left(h^{3}\right),
$$

and from the response of the retarded Green's function and then analytically continuing to Euclidean space, we finally obtain the Kubo relation for $\xi_{3}$ :

$$
\xi_{3}=3 \xi_{5}-\xi_{6}+\left(c_{s}^{2}-\frac{1}{3}\right)\left(\frac{\lambda_{3}}{2}-\kappa^{*}\right)+c_{s}^{2} \lim _{p_{z}, q_{z} \rightarrow 0} \frac{\partial^{2}}{\partial p_{z} \partial q_{z}} G_{E}^{t y, t y, \mu \mu}(p, q) .
$$




\section{A.4 Kubo relation for $\lambda_{4}$ and $\xi_{4}$}

The coefficients $\lambda_{4}$ and $\xi_{4}$ arise when the entropy, and therefore temperature, vary in space. For this to occur in equilibrium, the gravitational potential $h_{t t}$ must vary in space. Therefore we consider perturbations of $h_{t t}(x, y)$ to second order. The off-diagonal component of the stress-tensor, $\left\langle T^{x y}\right\rangle$, is

$$
\pi^{x y}=\pi_{h}^{x y}-\frac{\kappa}{2} h_{t t} \frac{\partial^{2} h_{t t}}{\partial x \partial y}+\kappa^{*} h_{t t} \frac{\partial h_{t t}}{\partial x \partial y}+\left(\frac{\lambda_{4}}{4 c_{s}^{4}}-\frac{\kappa}{4}+\frac{\kappa^{*}}{2}\right) \frac{\partial h_{t t}}{\partial x} \frac{\partial h_{t t}}{\partial y}
$$

that after analytic continuation reduces to

$$
\lambda_{4}=-\frac{c_{s}^{4}}{2} \lim _{p_{x}, q_{y} \rightarrow 0} \frac{\partial^{2}}{\partial p_{x} \partial q_{y}} G_{E}^{t t, t t, x y}(p, q)-2 \kappa^{*}+\kappa .
$$

To find $\xi_{4}$ we evaluate $\left\langle T_{\mu}^{\mu}\right\rangle$ for a perturbation of $h_{t t}(z)$. ¿From Eq. (A.5), we find the pressure through Eq. (A.9). We have $\Gamma_{t z}^{t}=-1 / 2 \partial_{z} h_{t t}, \Gamma_{t t}^{z}=-1 / 2 \partial_{z} h$, and $R_{t t}=-1 / 2 \partial_{z}^{2} h_{t t}-1 / 4\left(\partial_{z} h_{t t}\right)^{2}$, which result in

$$
(\epsilon+P)=(\bar{\epsilon}+\bar{P})+\left(1+\frac{1}{c_{s}^{2}}\right)\left(\frac{\bar{\epsilon}+\bar{P}}{2} h_{t t}+\left(\frac{\xi_{6}}{2}-\frac{2 \kappa^{*}}{3}+\frac{\kappa}{3}-\xi_{5}\right) \frac{\partial^{2} h_{t t}}{\partial z^{2}}\right)
$$

from Eq. (A.21), and

$$
\begin{aligned}
(\epsilon+P) R_{\sigma \mu} \bar{u}^{\sigma} \bar{u}^{\mu} & =-\frac{(\bar{\epsilon}+\bar{P})}{4}\left(\frac{\partial h_{t t}}{\partial z}\right)^{2}-\frac{(\epsilon+P)}{2} \frac{\partial^{2} h_{t t}}{\partial z^{2}} \\
R_{\sigma \mu} \bar{u}^{\sigma} \bar{u}^{\mu} \Pi= & -\frac{1}{2} \frac{\partial^{2} h_{t t}}{\partial z^{2}}\left(\xi_{5}-\frac{\xi_{6}}{2}\right) \frac{\partial^{2} h_{t t}}{\partial z^{2}} \\
\chi= & \chi_{h}+\frac{\bar{\epsilon}+\bar{P}}{4}\left(\frac{\partial h_{t t}}{\partial z}\right)^{2}-\frac{\partial(\epsilon+P)_{h}}{\partial z} \frac{\partial h_{t t}}{\partial z} \\
\Pi^{z z}= & \Pi_{h}^{z z}+\left(\frac{\xi_{4}}{4 c_{s}^{4}}-\frac{\xi_{6}}{4}+\frac{3 \xi_{5}+2 \kappa^{*}-\kappa}{6}+\frac{\lambda_{4}}{6 c_{s}^{4}}\right)\left(\frac{\partial h_{t t}}{\partial z}\right)^{2} \\
& +\left(\xi_{5}-\frac{\xi_{6}}{2}+\frac{2 \kappa^{*}-\kappa}{3}\right) h_{t t} \frac{\partial^{2} h_{t t}}{\partial z^{2}}
\end{aligned}
$$

After Fourier transforming and straightforward simplifications, the pressure reads

$$
\begin{aligned}
P_{h^{2}}= & \frac{\bar{\epsilon}+\bar{P}}{4}+\frac{\bar{\epsilon}+\bar{P}}{8}\left(1+\frac{1}{c_{s}^{2}}\right)+\frac{p_{z} q_{z}}{4}\left(\frac{2 \kappa^{*}}{3}+\frac{2 \lambda_{4}}{3 c_{s}^{4}}-\frac{\xi_{6}}{2}-\frac{\kappa}{3}+\xi_{5}+\frac{\xi_{4}}{c_{s}^{4}}\right) \\
& -\frac{p_{z} q_{z}}{4}\left(1+\frac{1}{c_{s}^{2}}\right)\left(\frac{\xi_{6}}{2}-\frac{2 \kappa^{*}}{3}+\frac{\kappa}{3}-\xi_{5}\right)+\mathcal{O}\left(p^{2}, q^{2}\right) .
\end{aligned}
$$

Inserting the above expressions for $P$ and $\Pi$ from Eq. (A.42), finally we get

$$
\begin{aligned}
\xi_{4}= & -\frac{c_{s}^{6}}{2} \lim _{p_{z}, q_{z} \rightarrow 0} \frac{\partial^{2}}{\partial p_{z} \partial q_{z}} G_{E}^{t t, t t, \mu \mu}(p, q)+6 c_{s}^{6}\left(\frac{\xi_{6}}{2}-\xi_{5}\right) \\
& +4 c_{s}^{6}\left(3-\frac{1}{c_{s}^{2}}\right)\left(+\frac{1}{4}\left(\frac{2 \kappa^{*}}{3}+\frac{2 \lambda_{4}}{3 c_{s}^{4}}-\frac{\xi_{6}}{2}-\frac{\kappa}{3}+\xi_{5}+\frac{\xi_{4}}{c_{s}^{4}}\right)\right. \\
& \left.-\frac{1}{4}\left(1+\frac{1}{c_{s}^{2}}\right)\left(\frac{\xi_{6}}{2}-\frac{2 \kappa^{*}}{3}+\frac{\kappa}{3}-\xi_{5}\right)\right) .
\end{aligned}
$$




\section{References}

[1] K. Adcox et al. [PHENIX Collaboration], Nucl. Phys. A 757 (2005) 184; B. B. Back et al. [PHOBOS Collaboration], Nucl. Phys. A 757 (2005) 28; I. Arsene et al. [BRAHMS Collaboration], Nucl. Phys. A 757 (2005) 1; J. Adams et al. [STAR Collaboration], Nucl. Phys. A 757 (2005) 102.

[2] D. Teaney, J. Lauret and E. V. Shuryak, Phys. Rev. Lett. 86 (2001) 4783;

P. Huovinen, P. F. Kolb, U. W. Heinz, P. V. Ruuskanen and S. A. Voloshin, Phys. Lett. B 503 (2001) 58; P. F. Kolb, U. W. Heinz, P. Huovinen, K. J. Eskola and K. Tuominen, Nucl. Phys. A 696 (2001) 197; T. Hirano and K. Tsuda, Phys. Rev. C 66 (2002) 054905; P. F. Kolb and R. Rapp, Phys. Rev. C 67 (2003) 044903.

[3] G. Baym, H. Monien, C. J. Pethick and D. G. Ravenhall, Phys. Rev. Lett. 64, 1867 (1990).

[4] P. B. Arnold, G. D. Moore and L. G. Yaffe, JHEP 0305, 051 (2003)

[hep-ph/0302165].

[5] G. Policastro, D. T. Son and A. O. Starinets, Phys. Rev. Lett. 87, 081601 (2001) [hep-th/0104066].

[6] P. Kovtun, D. T. Son and A. O. Starinets, Phys. Rev. Lett. 94, 111601 (2005) [hep-th/0405231].

[7] S. Borsányi, G. Endrodi, Z. Fodor, A. Jakovác, S. D. Katz, S. Krieg, C. Ratti and K. K. Szábo, JHEP 1011, 077 (2010) [arXiv:1007.2580 [hep-lat]]. A. Bazavov, T. Bhattacharya, M. Cheng, N. H. Christ, C. DeTar, S. Ejiri, S. Gottlieb and R. Gupta et al., Phys. Rev. D 80, 014504 (2009) [arXiv:0903.4379 [hep-lat]].

[8] Y. Aoki, Z. Fodor, S. D. Katz and K. K. Szábo, JHEP 0601, 089 (2006) [hep-lat/0510084]. C. R. Allton, S. Ejiri, S. J. Hands, O. Kaczmarek, F. Karsch, E. Laermann and C. Schmidt, Phys. Rev. D 68, 014507 (2003) [hep-lat/0305007]. C. R. Allton, S. Ejiri, S. J. Hands, O. Kaczmarek, F. Karsch, E. Laermann, C. Schmidt and L. Scorzato, Phys. Rev. D 66, 074507 (2002) [hep-lat/0204010]. F. Karsch, E. Laermann and A. Peikert, Phys. Lett. B 478, 447 (2000) [hep-lat/0002003].

[9] F. Karsch, S. Ejiri and K. Redlich, Nucl. Phys. A 774, 619 (2006) [hep-ph/0510126].

[10] S. Bhattacharyya, arXiv:1201.4654 [hep-th].

[11] K. Jensen, M. Kaminski, P. Kovtun, R. Meyer, A. Ritz and A. Yarom, Phys. Rev. Lett. 109, 101601 (2012) [arXiv:1203.3556 [hep-th]].

[12] N. Banerjee, J. Bhattacharya, S. Bhattacharyya, S. Jain, S. Minwalla and T. Sharma, arXiv:1203.3544 [hep-th].

[13] P. Romatschke, Class. Quant. Grav. 27, 025006 (2010).

[14] R. Baier, P. Romatschke, D. Son, A. Starinets and M. Stephanov, JHEP 0804, 100 (2008). 
[15] C. Schaefer, "Computation of the 2nd order transport coefficient $\kappa$ in the gluon plasma," poster presented at Strong and Electroweak Matter 2012, Swansea University, Swansea, United Kingdom.

[16] P. Romatschke, Int. J. Mod. Phys. E 19, 1 (2010) [arXiv:0902.3663 [hep-ph]].

[17] D. A. Teaney, arXiv:0905.2433 [nucl-th].

[18] G. Aarts and J. M. Martinez Resco, Nucl. Phys. Proc. Suppl. 119, 505 (2003) [hep-lat/0209033]. G. Aarts and J. M. Martinez Resco, JHEP 0204, 053 (2002) [hep-ph/0203177].

[19] H. B. Meyer, Prog. Theor. Phys. Suppl. 174, 220 (2008) [arXiv:0805.4567 [hep-lat]]. H. B. Meyer, Nucl. Phys. A 830, 641C (2009) [arXiv:0907.4095 [hep-lat]]. H. B. Meyer, Eur. Phys. J. A 47, 86 (2011) [arXiv:1104.3708 [hep-lat]].

[20] Y. Burnier and M. Laine, Eur. Phys. J. C 72, 1902 (2012) [arXiv:1201.1994 [hep-lat]].

[21] J. Schukraft, arXiv:1112.0550 [hep-ex]. S. Esumi [PHENIX Collaboration], J. Phys. G G 38, 124010 (2011) [arXiv:1110.3223 [nucl-ex]]. R. Lacey [for the PHENIX Collaboration], J. Phys. G G 38, 124048 (2011) [arXiv:1108.0457 [nucl-ex]]. H. Agakishiev et al. [STAR Collaboration], Phys. Lett. B 704, 467 (2011) [arXiv:1106.4334 [nucl-ex]]. J. D. de Deus, A. S. Hirsch, C. Pajares, R. P. Scharenberg and B. K. Srivastava, arXiv:1106.4271 [nucl-ex]. A. Dainese [ALICE Collaboration], arXiv:1106.1341 [nucl-ex].

[22] G. D. Moore and K. A. Sohrabi, Phys. Rev. Lett. 106, 122302 (2011) [arXiv:1007.5333 [hep-ph]].

[23] P. Romatschke and D. T. Son, Phys. Rev. D 80, 065021 (2009).

[24] L. E. Parker and D. J. Toms, Quantum Field Theory in Curved Spacetime.

[25] B. S. DeWitt, The global approach to quantum field theory. Vol. 1, 2, Int. Ser. Monogr. Phys. 114, 1 (2003).

[26] M. Lüscher, arXiv:1002.4232 [hep-lat].

[27] S. Caron-Huot, Phys. Rev. D 79, 125009 (2009) [arXiv:0903.3958 [hep-ph]].

[28] F. Karsch, Nucl. Phys. B 205, 285 (1982).

[29] S. Caracciolo, G. Curci, P. Menotti and A. Pelissetto, Annals Phys. 197, 119 (1990).

[30] T. R. Klassen, Nucl. Phys. B 533 (1998) 557 [hep-lat/9803010].

[31] J. Engels, F. Karsch and T. Scheideler, Nucl. Phys. B 564, 303 (2000) [hep-lat/9905002].

[32] P. Arnold, D. Vaman, C. Wu and W. Xiao, JHEP 1110, 033 (2011) [arXiv:1105.4645 [hep-th]].

[33] H. B. Meyer, Phys. Rev. D 76, 101701 (2007) [arXiv:0704.1801 [hep-lat]].

[34] H. B. Meyer, Nucl. Phys. B 795, 230 (2008) [arXiv:0711.0738 [hep-lat]]. 
[35] S. Bhattacharyya, V. E. Hubeny, S. Minwalla and M. Rangamani, JHEP 0802, 045 (2008).

[36] O. Saremi and K. A. Sohrabi, JHEP 1111, 147 (2011) [arXiv:1105.4870 [hep-th]].

[37] T. Banks and A. Zaks, Nucl. Phys. B 196, 189 (1982).

[38] A. Campos and E. Verdaguer, Phys. Rev. D 49, 1861 (1994) [gr-qc/9307027]. 